\title{
Thermocapillary migration of nondeformable drops
}

\author{
Z. Yin, ${ }^{1, a)}$ P. Gao, ${ }^{2, b)}$ W. Hu, ${ }^{1, c)}$ and L. Chang ${ }^{1, d)}$ \\ ${ }^{1}$ National Microgravity Laboratory, Institute of Mechanics, Chinese Academy of Sciences, \\ Beijing 100190, People's Republic of China \\ ${ }^{2}$ Department of Building Science, School of Architecture, Tsinghua University, Beijing 100084, \\ People's Republic of China
}

(Received 31 January 2008; accepted 1 July 2008; published online 7 August 2008)

\begin{abstract}
In this paper, we present a numerical study on the thermocapillary migration of drops. The NavierStokes equations coupled with the energy conservation equation are solved by the finite-difference front-tracking scheme. The axisymmetric model is adopted in our simulations, and the drops are assumed to be perfectly spherical and nondeformable. The benchmark simulation starts from the classical initial condition with a uniform temperature gradient. The detailed discussions and physical explanations of migration phenomena are presented for the different values of (1) the Marangoni numbers and Reynolds numbers of continuous phases and drops and (2) the ratios of drop densities and specific heats to those of continuous phases. It is found that fairly large Marangoni numbers may lead to fluctuations in drop velocities at the beginning part of simulations. Finally, we also discuss the influence of initial conditions on the thermocapillary migrations. () 2008 American Institute of Physics. [DOI: 10.1063/1.2965549]
\end{abstract}

\section{INTRODUCTION}

The migration phenomena of bubbles or droplets in liquids are very interesting topics in material science, chemical engineering, and fundamental research. Under the zerogravity condition, if the temperature gradient of the background fluid is nonzero, bubbles or droplets will move because the variance of interfacial tension on the drop can produce a shear stress on both sides of the interface. As a result, the bubbles/drops will move from the cold side to the warm side of the background fluid. Such motion is termed thermocapillary migration.

Throughout this paper, symbols with subscript 1 denote the physical coefficients of the continuous phase and those with subscript 2 are the values of the bubble/drop. The interfacial tension is normally treated as a linearly decreasing function of temperature,

$$
\sigma=\sigma_{0}+\sigma_{T}\left(T_{0}-T\right),
$$

where $\sigma_{0}$ is the interface tension at a reference temperature $T_{0}$, and

$$
\sigma_{T}=-(d \sigma / d T)=\text { const. }
$$

The reference velocity $U$ for the motion, obtained by balancing the thermocapillary stress on the interface of the bubble/ drop with the viscous stress, is defined as

$$
U=\left|\sigma_{T}\right| \nabla T R / \mu_{1},
$$

where $R$ is the radius of the bubble/drop, $\nabla T$ the temperature gradient imposed on the external fluid, and $\mu$ the dynamic viscosity. Besides the fluid densities, the most important fac-

\footnotetext{
a) Author to whom correspondence should be addressed. Electronic mail: zhaohua.yin@imech.ac.cn.

${ }^{b)}$ Electronic mail: gaopeng@mail.tsinghua.edu.cn.

${ }^{c}$ Electronic mail: wrhu@imech.ac.cn.

${ }^{\mathrm{d})}$ Electronic mail: changlei@imech.ac.cn.
}

tors in the thermocapillary migration are the Reynolds numbers $(\mathrm{Re})$ and Marangoni numbers (Ma) of the bubble/drop and the continuous phase. We have the following six nondimensional numbers characterizing the problem:

$$
\begin{aligned}
& \operatorname{Re}_{1}=\frac{R U}{\nu_{1}}, \\
& \mathrm{Ma}_{1}=\frac{R U}{\kappa_{1}}, \\
& \alpha=\frac{\mathrm{Re}_{1}}{\mathrm{Re}_{2}}=\frac{\nu_{2}}{\nu_{1}}, \\
& \beta=\frac{\mathrm{Ma}_{1}}{\mathrm{Ma}_{2}}=\frac{\kappa_{2}}{\kappa_{1}}, \\
& \xi=\frac{\rho_{2}}{\rho_{1}}, \\
& \gamma=\frac{C_{p 2}}{C_{p 1}},
\end{aligned}
$$

where $\nu, \kappa, \rho$, and $C_{p}$ represent the kinematic viscosity, the thermal diffusivity, the density, and the specific heat, respectively. Moreover, an important relation on some of the parameters above is given as ${ }^{1}$

$$
\kappa=\frac{k_{H}}{\rho C_{p}},
$$

where $k_{H}$ is the thermal conductivity.

The first study on the thermocapillary migration of bubbles and droplets was carried out by Young et al. ${ }^{2}$ [referred to as the Young-Goldstein-Block (YGB) theory]. In their work, $\operatorname{Re}_{1}$ and $\mathrm{Ma}_{1}$ are sufficiently small compared to 


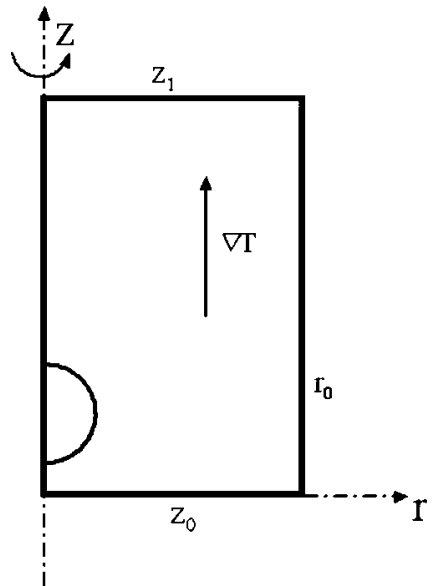

FIG. 1. The schematic diagram of the axisymmetric model for the Marangoni migration.

unity, and hence the inertial terms in the Navier-Stokes equations and the convective transport terms in the energy equation can be neglected. The migration velocity of the drop in the YGB theory is

$$
V_{\mathrm{YGB}}=\frac{2 U}{\left(2+3 \mu_{2} / \mu_{1}\right)\left(2+k_{H 2} / k_{H 1}\right)} .
$$

Later, using the matched asymptotic method, Subramanian $^{3,4}$ extended the YGB theory to high-order result for small $\mathrm{Ma}_{1}$ when $\mathrm{Re}_{1}=0$ and found that the correction to the result Eq. (8) is zero at $O\left(\mathrm{Ma}_{1}\right)$ and the first nonzero correction appears at $O\left(\mathrm{Ma}_{1}^{2}\right)$. In addition, they concluded that the heat convection may reduce the scaled migration velocity of the bubble. Balasubramaniam ${ }^{5}$ analyzed the situation of a gas bubble when $\operatorname{Re}_{1}$ is zero or infinite and extended these results to situations of large values of $\mathrm{Ma}_{1}$. Balasubramaniam and Subramanian ${ }^{6}$ studied the effect of the convection inside the drop with large $\mathrm{Ma}_{1}$ and found that the variation of temperature along the drop surface leads to a linear increase in drop migration velocity when the value of $\mathrm{Ma}_{1}$ increases.

Since the work of YGB, numerous experiments on thermocapillary migrations were performed on the ground. Most of them, with relatively large drops, have been conducted on liquid pairs of nearly matched densities to reduce the effect of gravity. ${ }^{7-9} \mathrm{Xie}$ et al. performed a microgravity shaft experimental study of a drop Marangoni migration at intermediate $\operatorname{Re}_{1}$ of $O(10) .^{10}$ They found that the drop migration velocities are much smaller than those of the YGB theory. On the fourth Shen-Zhou spaceship, Xie et al. carried out experiments with larger values of $\mathrm{Re}_{1}$ (up to 300) and $\mathrm{Ma}_{1}$ (up to 5500). ${ }^{11}$

There are also lots of numerical studies on this subject. Szymczyk and Siekmann ${ }^{12}$ first calculated the steady migration of bubbles. Shankar and Subramanian ${ }^{13}$ used the velocity field of the Stokes solution in energy equation, with values of $\mathrm{Ma}_{1}$ up to 200. Balasubramaniam and Lavery ${ }^{14}$ simulated bubble migrations in the cases of $\operatorname{Re}_{1}$ $\in\left[10^{-7}, 2000\right]$ and $\mathrm{Ma}_{1} \in\left[10^{-7}, 1000\right]$, and they concluded that Marangoni numbers have larger influence on the migra-

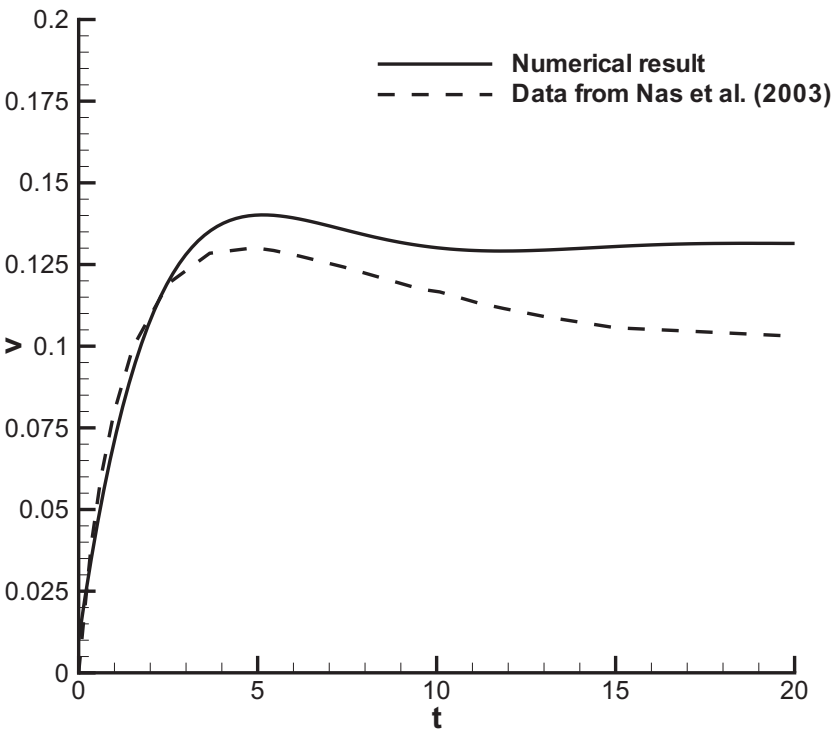

FIG. 2. Time evolutions of drop migration velocity and their comparison with Nas (Ref. 17). Here, $\mathrm{Re}_{1}=5, \mathrm{Ma}_{1}=20, \alpha=0.5, \beta=2.0, \xi=0.5$, and $\gamma=0.5$.

tion velocity than Reynolds numbers do. The extended work of Ref. 14 revealed that the scaled velocity of a liquid drop initially decreases as $\mathrm{Ma}_{1}$ increases, but increases after $\mathrm{Ma}_{1}$ reaches a certain value (around 50-200). Note that their simulations were performed chiefly for certain experimental systems. ${ }^{15}$ Unlike our analysis in this paper, their discussions on values of $\mathrm{Ma}_{1}$ were based on the fixing of the experimental materials instead of the fixing of the other five nondimensional numbers.

Numerical simulations on the three-dimensional thermocapillary motion of deformable viscous drops were reported by Haj-Harir et al. ${ }^{16}$ They used the level-set method to catch the interface and found that the strong heat convection may retard the thermocapillary motion of the drop be-

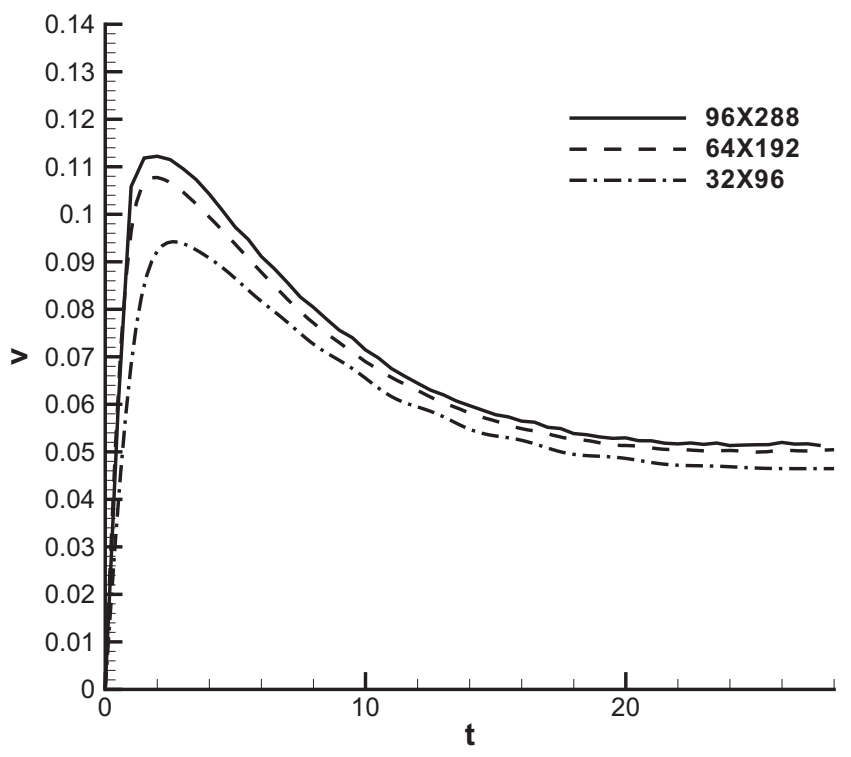

FIG. 3. The migration velocity of drop plotted vs time for three grids of $96 \times 288,64 \times 192$, and $32 \times 96 ; \mathrm{Ma}_{1}=100, \mathrm{Re}_{1}=1, \alpha=1, \beta=0.5, \xi=1$, and $\gamma=1$. 


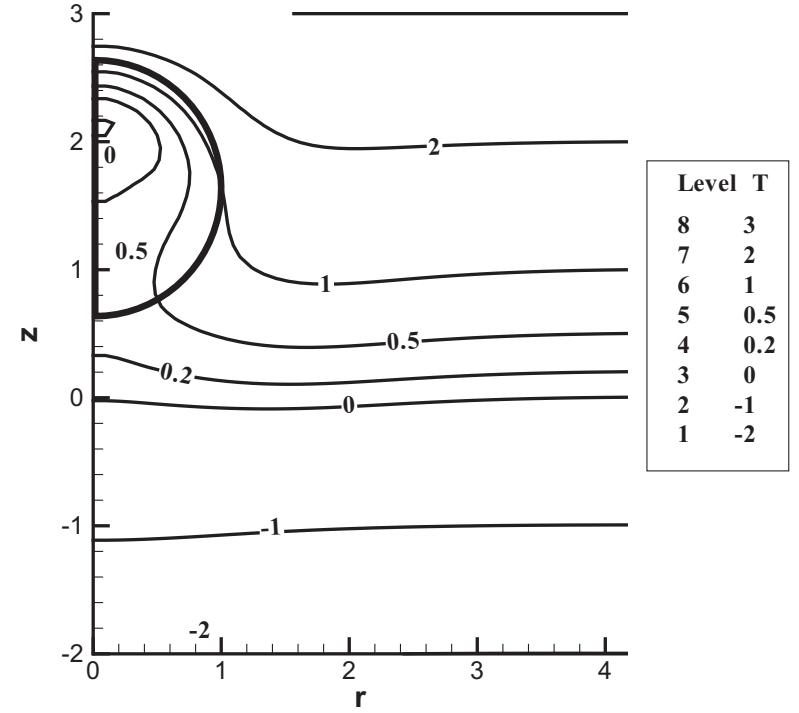

(a)

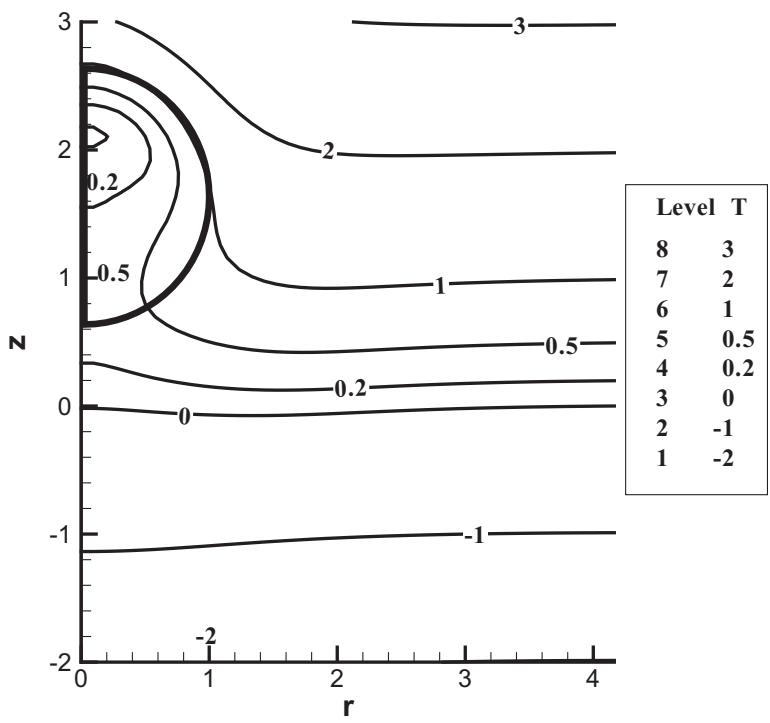

(c)

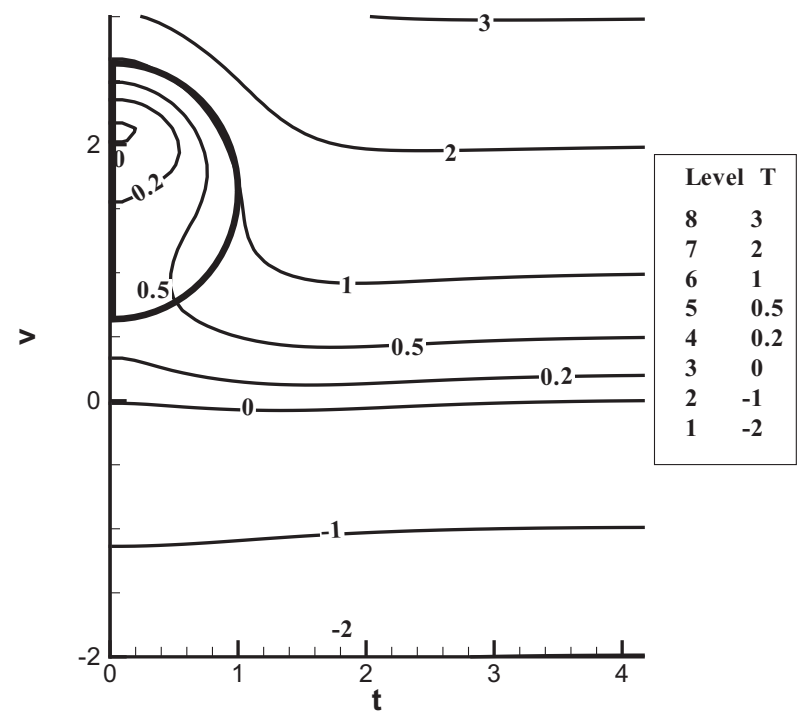

(b)

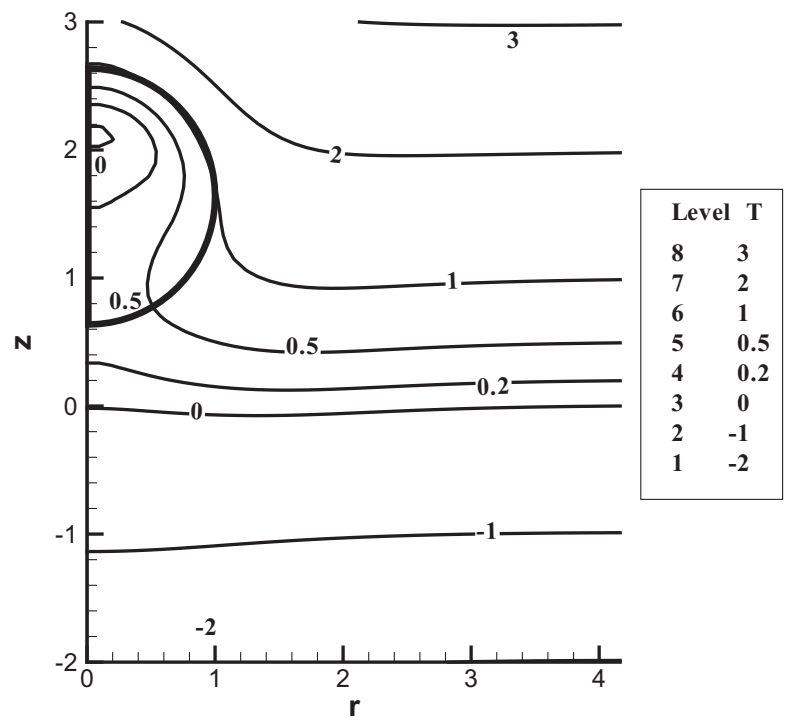

(d)

FIG. 4. The comparison of temperature fields between results from different computational domains at $T=26$. Here, $\operatorname{Ma}_{1}=100, \operatorname{Re}_{1}=1, \alpha=1, \beta=0.5, \xi=1$, and $\gamma=1$. The sizes of domains are (a) $6 R \times 6 R$; (b) $6 R \times 12 R$; (c) $6 R \times 18 R$; (d) $6 R \times 36 R$.

cause the isotherms get wrapped around the front surface of the drop and reduce the surface temperature gradient. $\mathrm{Nas}^{17,18}$ calculated the thermocapillary interaction of multidrops with the front-tracking finite-difference methods.

Despite the efforts mentioned above, it is still unclear what kinds of phenomena might happen when different sets of nondimensional numbers are adopted, and this will be the focus of this paper. In addition, we restrict our discussions within the scope of nondeformable drops because even the deformation of the large drop is negligible. ${ }^{19,20}$ Section II describes the governing equations and numerical schemes, respectively. In Sec. III, starting from a traditional initial condition in this field, we present a benchmark simulation based on which we carry on further discussions. In Sec. IV, we outline the migration phenomena of drops with different sets of nondimensional numbers. Finally, the influences of initial conditions are discussed in Sec. V.

\section{GOVERNING EQUATIONS AND NUMERICAL METHODS}

In our investigation, the spherical drop is surrounded by the continuous phase in a cylindrical container. As shown in Fig. 1, the symmetric axis of the container is taken as the $z$-axis in a cylindrical coordinate system, and the temperature varies linearly in the $z$-direction initially. The drop migrates from the rest and is assumed to keep its spherical shape 
during the whole process. The governing equations of the problem under the zero-gravity condition can be written as ${ }^{1,21,22}$

$$
\begin{aligned}
& \nabla \cdot \mathbf{u}=0, \\
& \frac{\partial(\rho \mathbf{u})}{\partial t}+\nabla \cdot(\rho \mathbf{u u})=-\nabla p+\nabla \cdot \mu\left(\nabla \mathbf{u}+\nabla^{T} \mathbf{u}\right)-\mathbf{F}^{\sigma}, \\
& \rho C_{p}\left[\frac{\partial(T)}{\partial t}+\nabla \cdot(\mathbf{u} T)\right]=\nabla \cdot\left(k_{H} \nabla T\right),
\end{aligned}
$$

where $p$ represents the pressure, $\mathbf{u}$ or $(u, w)$ the velocity vector, and $T$ the temperature. $\mathbf{F}^{\sigma}$ is the integral over the interface of the drop $(S)$, which results in a force that is smooth and continuous along the interface,

$$
\mathbf{F}^{\sigma}=\int_{S} \sigma \zeta \mathbf{n} \delta\left(\mathbf{x}-\mathbf{x}_{\text {interface }}\right) d S \cdot \mathbf{t},
$$

where $\zeta$ is twice the mean curvature, $\delta$ a three-dimensional delta function, $\mathbf{n}$ the interface unit normal vector pointing outward from the drop, and $\mathbf{t}$ the unit tangent vector.

The nondimensional quantities are defined as

$$
\begin{aligned}
& \overline{\mathbf{u}}=\mathbf{u} / U, \quad \bar{r}=r / R, \quad \bar{z}=z / R, \\
& \bar{t}=t /\left(\frac{R}{U}\right), \quad \bar{p}=p /\left(\rho_{1} U^{2}\right), \quad \bar{T}=T /(|\nabla T| R), \\
& \bar{\rho}=\rho / \rho_{1}, \quad \bar{\mu}=\mu / \mu_{1}, \quad \overline{k_{H}}=k_{H} / k_{H 1}, \\
& \overline{C_{p}}=C_{p} / C_{p 1}, \quad \overline{\mathbf{F}}^{\sigma}=\mathbf{F}^{\sigma} R /\left(\rho_{1} U^{2}\right) .
\end{aligned}
$$

Thus we have the full nondimensional equations in our numerical simulations,

$$
\begin{aligned}
\frac{\partial \bar{u}}{\partial \bar{r}}+\frac{\bar{u}}{\bar{r}}+\frac{\partial \bar{w}}{\partial \bar{z}}=0, & \\
\frac{\partial(\bar{\rho} \bar{u})}{\partial \bar{t}}+ & \bar{u} \frac{\partial(\bar{\rho} \bar{u})}{\partial \bar{r}}+\bar{w} \frac{\partial(\bar{\rho} \bar{u})}{\partial \bar{z}} \\
= & -\frac{\partial \bar{p}}{\partial \bar{r}}+\frac{1}{\operatorname{Re}_{1}}\left(\bar{\mu}\left\{\frac{\partial}{\partial \bar{r}}\left[\frac{1}{\bar{r}} \frac{\partial(\bar{r} \bar{u})}{\partial \bar{r}}\right]+\frac{\partial^{2} \bar{u}}{\partial \bar{z}^{2}}\right\}+\frac{\partial \bar{\mu}}{\partial \bar{r}}\left(\frac{\partial \bar{u}}{\partial \bar{r}}+\frac{\partial \bar{u}}{\partial \bar{r}}\right)\right. \\
& \left.+\frac{\partial \bar{\mu}}{\partial \bar{z}}\left(\frac{\partial \bar{w}}{\partial \bar{r}}+\frac{\partial \bar{u}}{\partial \bar{z}}\right)\right)-\left(\overline{\mathbf{F}}^{\sigma}\right)_{r},
\end{aligned}
$$$$
\frac{\partial(\bar{\rho} \bar{w})}{\partial \bar{t}}+\bar{u} \frac{\partial(\bar{\rho} \bar{w})}{\partial \bar{r}}+\bar{w} \frac{\partial(\bar{\rho} \bar{w})}{\partial \bar{z}}
$$$$
=-\frac{\partial \bar{p}}{\partial \bar{z}}+\frac{1}{\operatorname{Re}_{1}}\left\{\bar{\mu}\left[\frac{1}{\bar{r}} \frac{\partial}{\partial \bar{r}}\left(\bar{r} \frac{\partial \bar{w}}{\partial \bar{r}}\right)+\frac{\partial^{2} \bar{w}}{\partial \bar{z}^{2}}\right]+\frac{\partial \bar{\mu}}{\partial \bar{r}}\left(\frac{\partial \bar{w}}{\partial \bar{r}}+\frac{\partial \bar{u}}{\partial \bar{z}}\right)\right.
$$$$
\left.+\frac{\partial \bar{\mu}}{\partial \bar{z}}\left(\frac{\partial \bar{w}}{\partial \bar{z}}+\frac{\partial \bar{w}}{\partial \bar{z}}\right)\right\}-\left(\overline{\mathbf{F}}^{\sigma}\right)_{z}
$$

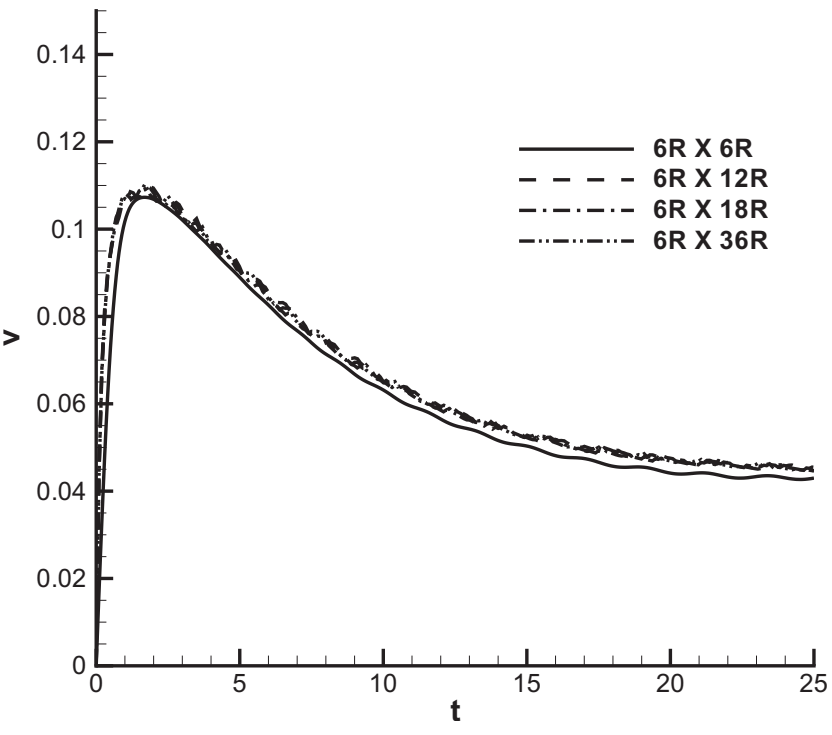

FIG. 5. Time evolutions of migration velocities between results from different computational domains $\left(\mathrm{Ma}_{1}=100, \mathrm{Re}_{1}=1, \alpha=1, \beta=0.5, \xi=1\right.$, and $\gamma=1)$.

$$
\begin{aligned}
\bar{\rho} \overline{C_{p}}\left(\frac{\partial \bar{T}}{\partial \bar{t}}+\bar{u} \frac{\partial \bar{T}}{\partial \bar{r}}+\bar{w} \frac{\partial \bar{T}}{\partial \bar{z}}\right)= & \frac{1}{\mathrm{Ma}_{1}}\left[\frac{1}{\bar{r}} \frac{\partial}{\partial \bar{r}}\left(\bar{r} \bar{k}_{H} \frac{\partial \bar{T}}{\partial \bar{r}}\right)\right. \\
& \left.+\frac{\partial}{\partial \bar{z}}\left(\overline{k_{H}} \frac{\partial \bar{T}}{\partial \bar{z}}\right)\right] .
\end{aligned}
$$

The boundaries except the symmetrical axis are treated as no-slip walls, and the temperature is fixed on the walls throughout the simulations,

$$
\begin{aligned}
& \left.\bar{T}\right|_{\bar{z}=z_{0} / R}=T_{0} /(|\nabla T| R)+z_{0} / R, \\
& \left.\bar{T}\right|_{\bar{z}=z_{1} / R}=T_{0} /(|\nabla T| R)+z_{1} / R, \\
& \left.\bar{T}\right|_{\bar{r}=r_{0} / R}=T_{0} /(|\nabla T| R)+z / R, \\
& \left.\bar{u}\right|_{\bar{z}=z_{0} / R}=\left.\bar{w}\right|_{\bar{z}=z_{0} / R}=0, \\
& \left.\bar{u}\right|_{\bar{z}=z_{1} / R}=\left.\bar{w}\right|_{\bar{z}=z_{1} / R}=0, \\
& \left.\bar{u}\right|_{\bar{r}=r_{0} / R}=\left.\bar{w}\right|_{\bar{r}=r_{0} / R}=0 .
\end{aligned}
$$

In the calculation, we always set the vertical position of initial drop center at $z=0$, and $T_{0}$ is the initial temperature of continuous phase at $z=0$. Notice that $T_{0}$ is not always the temperature of the drop center in this paper because some of our simulations (in Sec. V) do not use the classical initial conditions.

On the symmetric axis, we have

$$
\left.\frac{\partial \bar{T}}{\partial \bar{r}}\right|_{\bar{r}=0}=0,\left.\quad \bar{u}\right|_{\bar{r}=0}=0,\left.\quad \frac{\partial \bar{w}}{\partial \bar{r}}\right|_{\bar{r}=0}=0 .
$$

For most simulations except those in Sec. V, the classical initial conditions used in most literatures are adopted, 
(a)

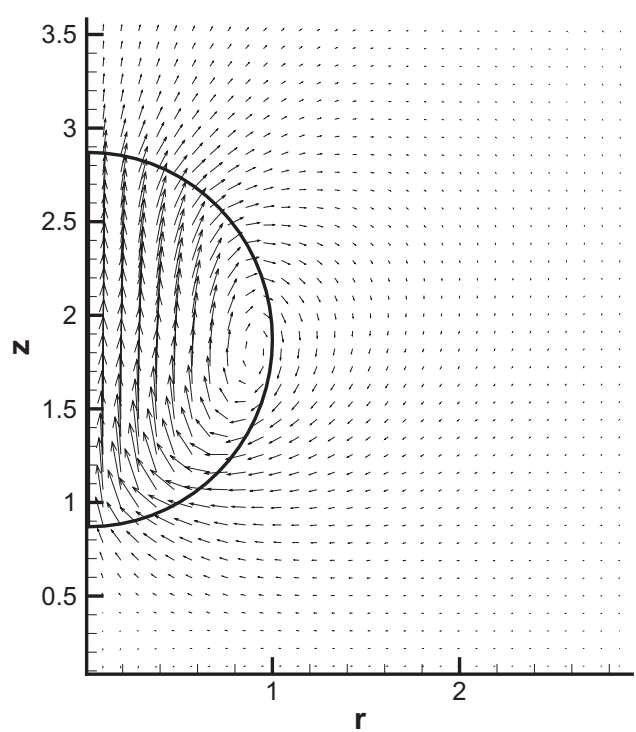

(b)

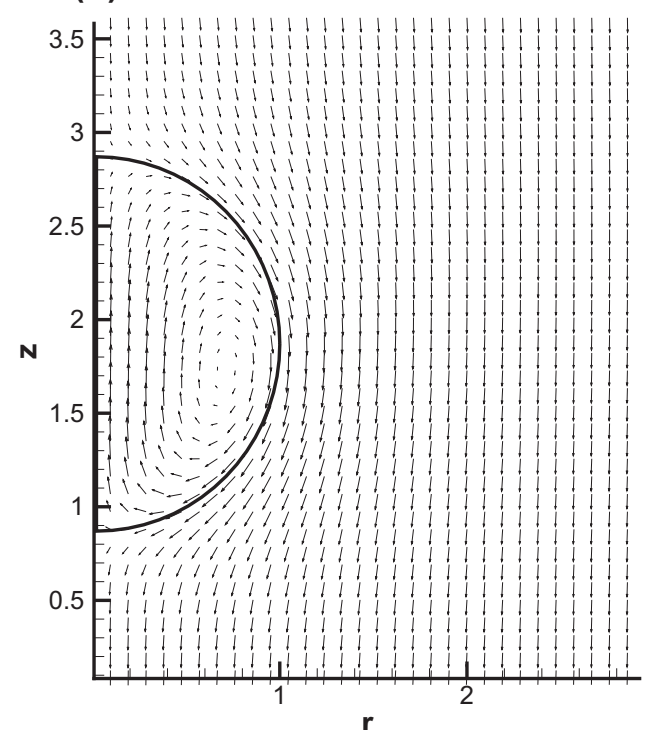

FIG. 6. A typical velocity field (a) in the laboratory reference frame and (b) in the reference frame moving with the drop.

$\left.\bar{u}\right|_{\bar{t}=0}=\left.\bar{w}\right|_{\bar{t}=0}=0$,

$$
\left.\bar{T}\right|_{\bar{t}=0}=T_{0} /(|\nabla T| R)+z / R .
$$

For simulations in Sec. V, initial temperature fields inside the drops are constant, while all other conditions (the initial tem- perature field outside of the drop and the initial velocity field of the entire domain) remain the same as the above.

In the following, symbols without bars above are adopted to denote nondimensional values. It should be mentioned that $\rho, \mu, C_{p}$, and $k_{H}$ are constants within each kind of fluids in the calculation, and that a smooth function is

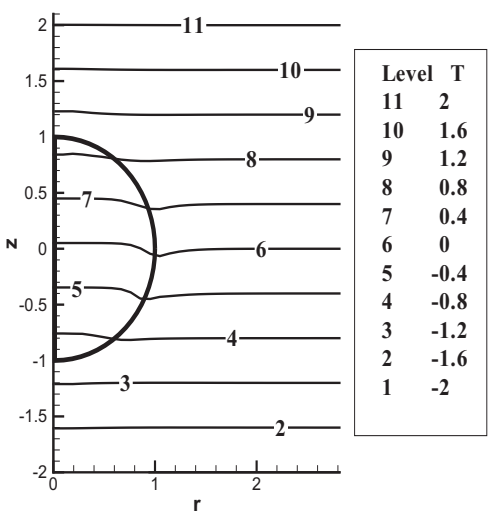

(a) $t=1$

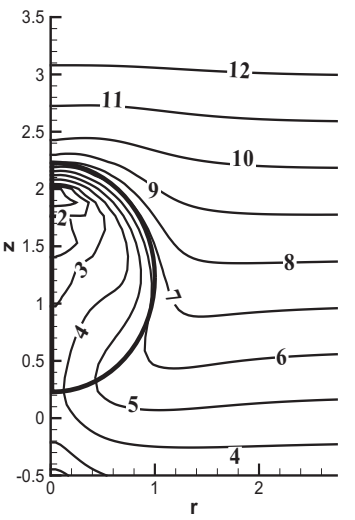

(d) $\mathrm{t}=14$

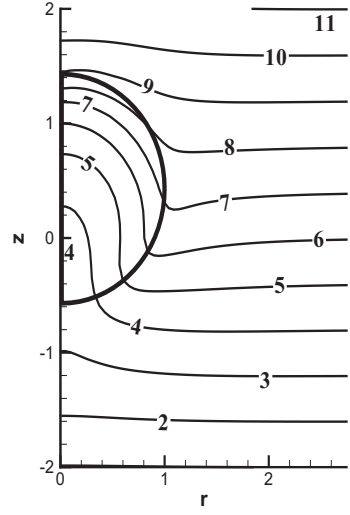

(b) $t=4$

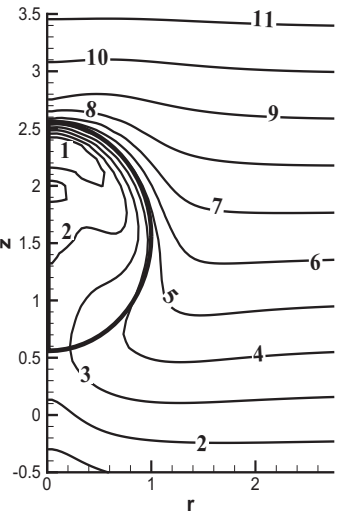

(e) $t=20$

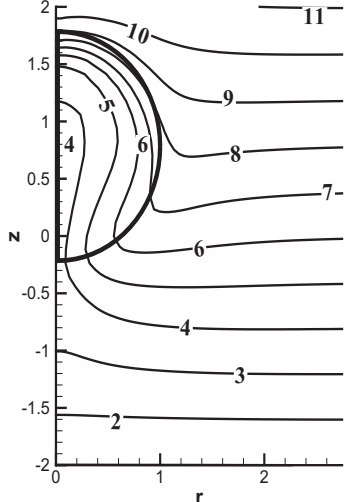

(c) $t=8$

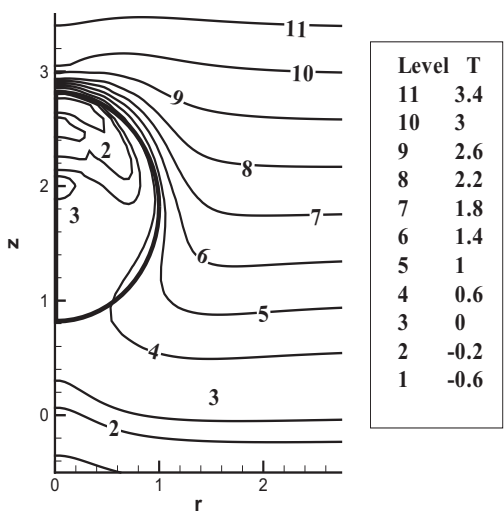

(f) $t=50$

FIG. 7. The isotherms of the drop from $t=1$ to $t=50 ; \mathrm{Ma}_{1}=500, \mathrm{Re}_{1}=1, \alpha=1, \beta=0.5, \xi=1$, and $\gamma=1$ (enhanced online). 


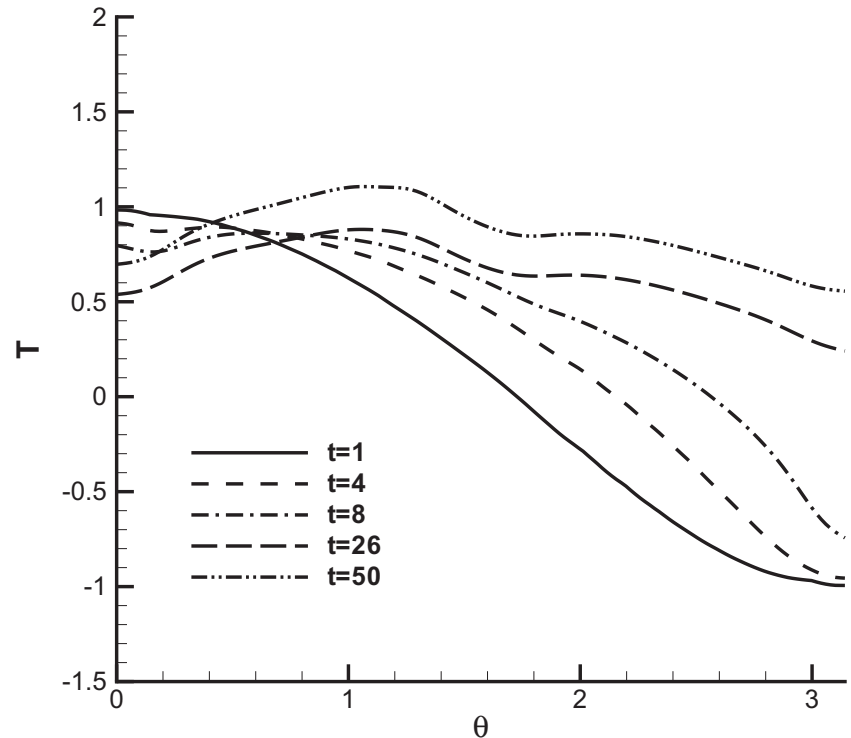

FIG. 8. Scaled temperature distribution along the drop interface for various time: $\mathrm{Ma}_{1}=500, \mathrm{Re}_{1}=1, \alpha=1, \beta=0.5, \xi=1$, and $\gamma=1 . \theta$ is defined as the angle from the interface to the drop center. $\theta=0$ corresponds to the top of the drop, and $\theta=\pi$ to the bottom of the drop.

adopted to reduce numerical oscillations along the interface (see Ref. 23 for more details).

We adopt the finite-difference method to solve the system above. The equations are discretized by second-order center-difference methods on fixed, regular, staggered Marker-and-Cell grids. The time integration adopts the explicit forward Euler scheme. The Navier-Stokes equations are solved by the projection method with the front-tracking method to catch the interface, ${ }^{24,25}$ and additional computational grids are introduced to explicitly mark the position of the interface. In the calculation, the interface tension is converted into a body force with an interpolation function, ${ }^{26}$ and the variables on the fixed grid can also be transferred onto the interface grid. More details of the numerical methods are presented in Ref. 23.

Our numerical solver yields results which fit the analytical outcomes of YGB in Ref. 23. To further validate our code, we compare our unsteady results to Nas's work. ${ }^{17}$ From Fig. 2, it can be observed that the trends on the migration velocity of two simulations are quite similar: a slight decrease in the velocities occurs before their reaching the steady values in both cases. In Nas's work, the deformation of the drop is considered; hence, the value of the migration velocity is slightly smaller than ours.

We also test our code when large Marangoni numbers are adopted on three resolutions. Figure 3 shows that the velocity curve seems to converge when grids become finer, and the difference between $96 \times 288$ and $64 \times 192$ is below $3 \%$.

Finally, it is essential to discuss the role of computational domains. In practice, simulations are always carried out on the smallest possible domains and with the lowest possible resolutions to save CPU hours. Therefore, it is interesting to know what kinds of differences will be caused by various domain sizes in this study.

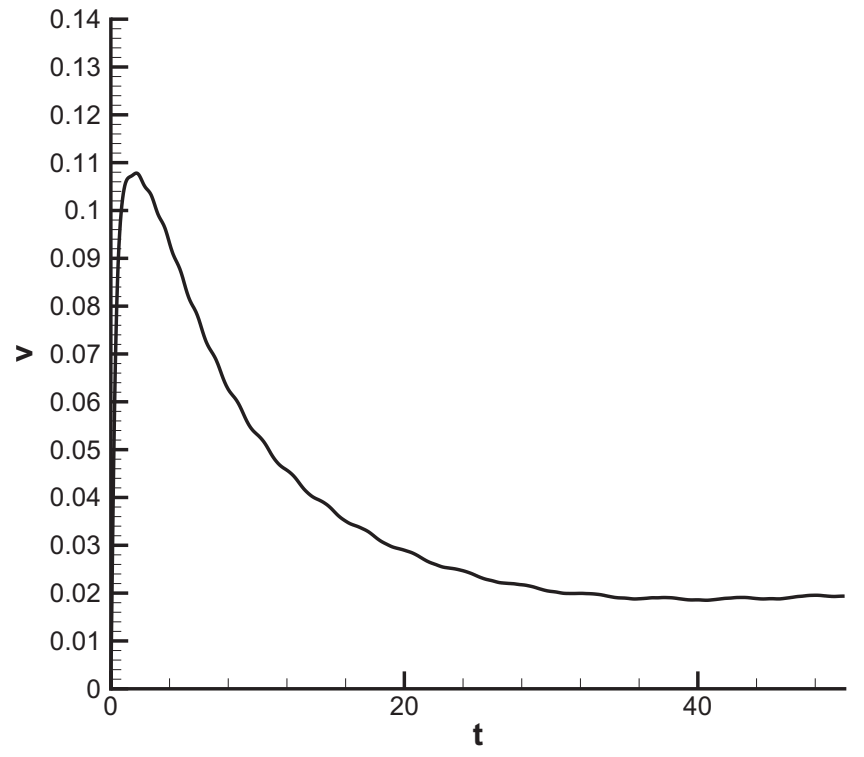

FIG. 9. Time evolutions of drop migration velocity as $\mathrm{Ma}_{1}=500, \mathrm{Re}_{1}=1$, $\alpha=1, \beta=0.5, \xi=1$, and $\gamma=1$.

We conducted simulations with the same set of parameters $\left(\mathrm{Ma}_{1}=100, \operatorname{Re}_{1}=1, \alpha=1, \beta=0.5, \xi=1\right.$, and $\left.\gamma=1\right)$ on four kinds of domains: $6 R \times 6 R, 6 R \times 12 R, 6 R \times 18 R$, and $6 R \times 36 R$. The resolution of each simulation is chosen to make the density of the grid unchanged when the domain size varies, so the resolutions of the four runs above are 64 $\times 64,64 \times 128,64 \times 192$, and $64 \times 384$, respectively. The resulting temperature fields in the four runs are almost identical (Fig. 4), except that the $T=1$ isotherm in $r<1$ region of Fig. 4(a) does not overlap with the interface as those of Figs. 4(b)-4(d). On the other hand, there is a noticeable difference in the migration speed plot (Fig. 5), and the velocity of the $6 R \times 6 R$ run is $5 \%$ lower than those of larger-domain runs.

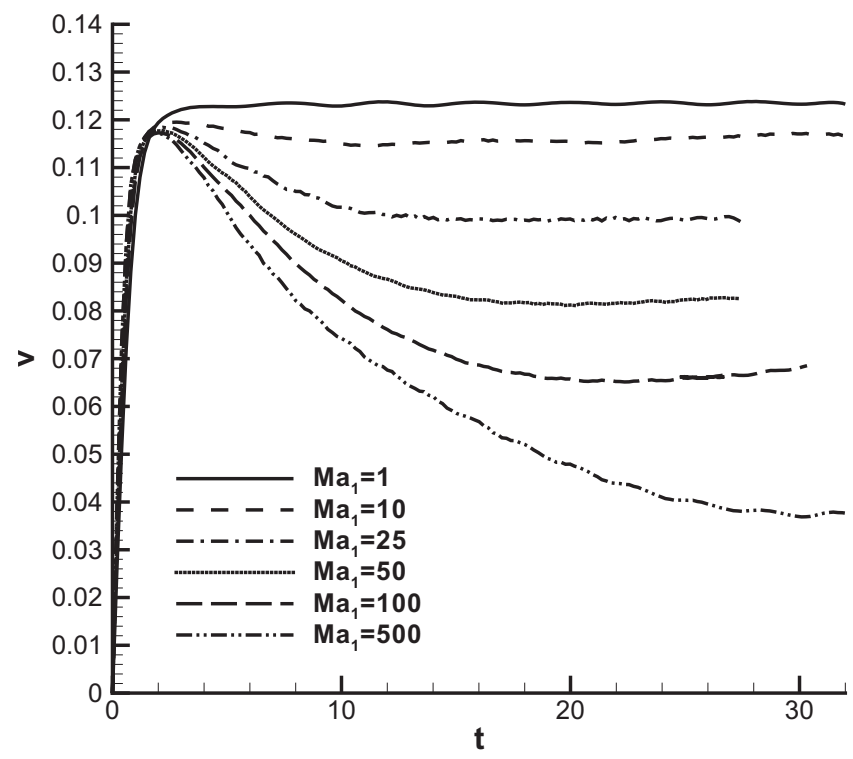

FIG. 10. Time evolutions of drop migration velocity with different values of $\mathrm{Ma}_{1}: \mathrm{Re}_{1}=1, \alpha=1$, and $\beta=1$. 


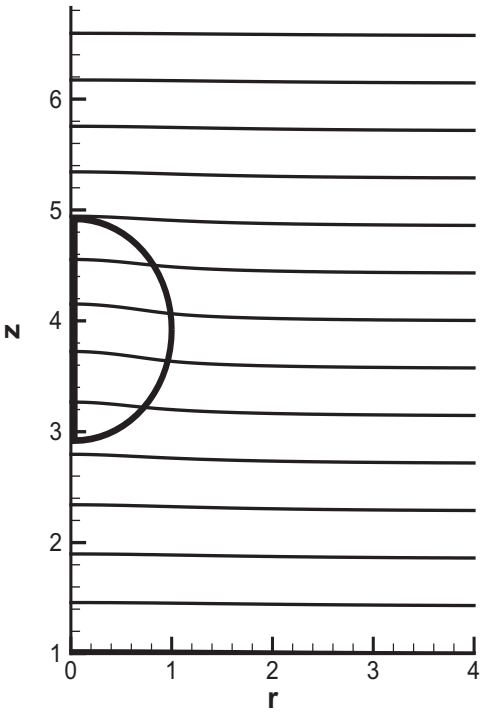

(a) $\mathrm{Ma}_{1}=1$

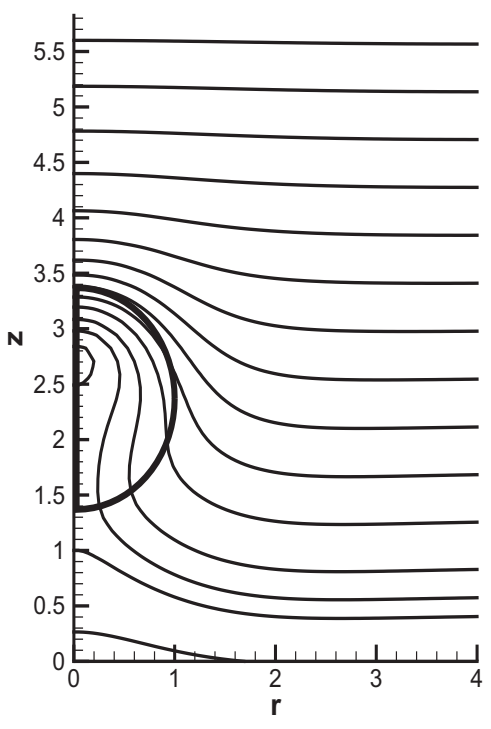

(d) $\mathrm{Ma}_{1}=50$

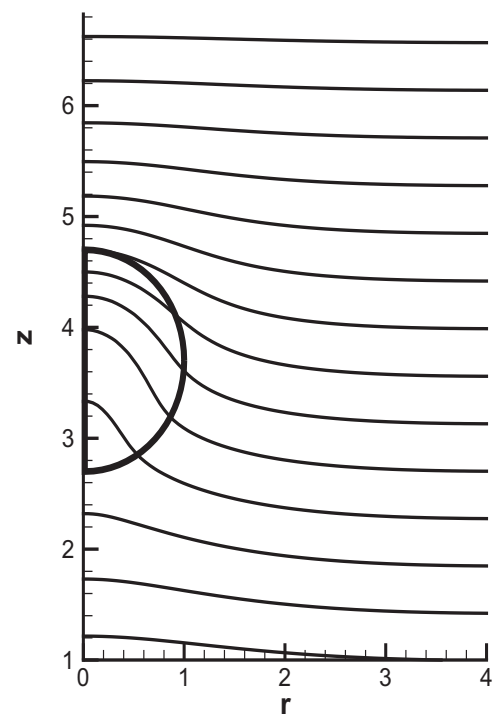

(b) $M a_{1}=10$

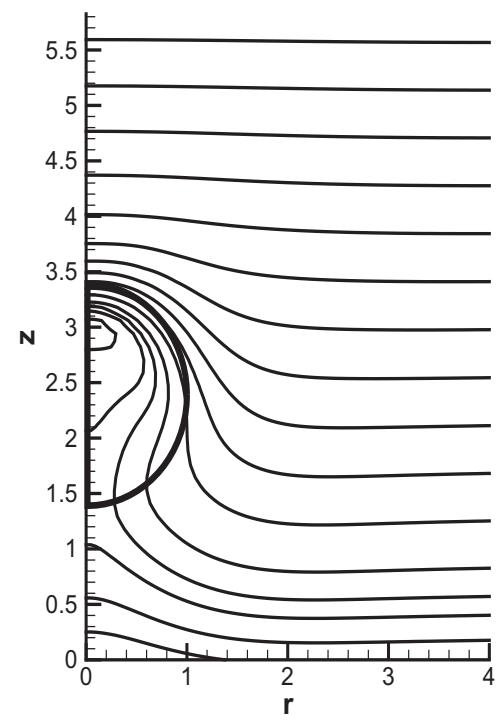

(e) $\mathrm{Ma}_{1}=100$

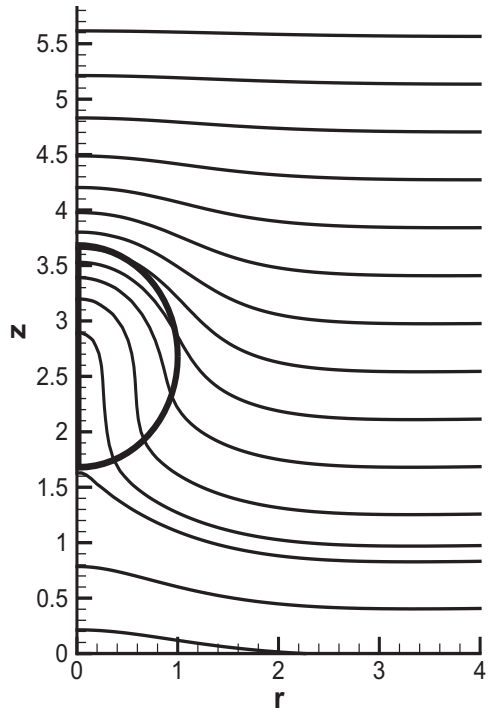

(c) $M a_{1}=25$

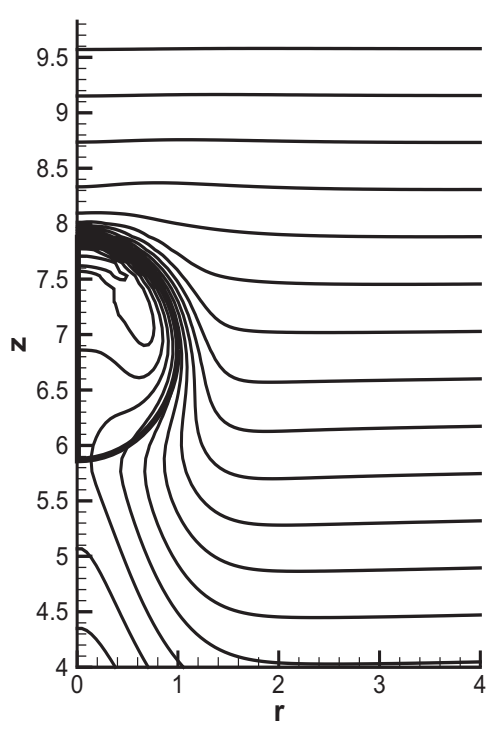

(f) $\mathrm{Ma}_{1}=500$

FIG. 11. The temperature field at $t=28$ for different values of $\mathrm{Ma}_{1}: \mathrm{Re}_{1}=1, \alpha=1$, and $\beta=1$ (enhanced online).

Throughout the paper, all computed domains, except those of the runs shown in Figs. 4 and 5 , are $6 R \times 18 R$, which should be large enough to avoid any boundary effect.

\section{A BENCHMARK SIMULATION AND BRIEF DISCUSSION ON ITS INITIAL CONDITION}

Throughout this study, the time step is 0.0005 and the resolution is $128 \times 384$. The initial drop center is $6 R$ above the bottom, which is fairly far away from the boundaries. The first objective of this study is to analyze the unsteady thermocapillary migration of an isolated viscous drop when the Marangoni number is large. In the meanwhile, the large Ma investigation will also serve as a benchmark to facilitate further discussions in the next two sections.

In the following simulation, we adopt $\mathrm{Ma}_{1}=500, \mathrm{Re}_{1}$ $=1, \alpha=1, \beta=0.5, \xi=1$, and $\gamma=1$. Once the simulation starts, the surface tension will drive the drop liquid near the interface from the warm side to the cold region. A pair of vortices are formed inside the drop, which can be seen from the velocity field in the reference frame moving with the drop [Fig. 6(b)].

To the problem when $\mathrm{Ma}$ is large, the influence of heat convection is more important than that of heat diffusion, and the velocity field is strongly coupled with the temperature field. So in order to understand the phenomena in the drop migration, it is very important to analyze the evolution of the temperature field. At the beginning of the migration, the temperature varies linearly in the $z$-direction from the cold wall at the bottom to the hot wall on the top [Fig. 7(a)]. The vortices inside the drop will disturb the initial temperature field, and the isotherms outside of the drop will bend downward near the interface [Figs. 7(b) and 7(c)]. If the heat con- 
vection effect is strong enough, the cold liquid inside the drop can reach the upside of the drop before being warmed up by its surroundings, and then move down along the interface. And the temperature field will form an "eye" pattern around $t=50$ [Figs. $7(\mathrm{~d})-7(\mathrm{f})$ ]. The entire process is even clearer in the movie associated with Fig. 7.

The variance of the temperature field will lead to the variance of the temperature on the interface. Figure 8 shows the evolution of scaled temperature distribution along the interface. In the beginning, the temperature curve is only related to the vertical position and varies as a cosine function of $\theta$ (the solid curve in Fig. 8). From $t=1$ to $t=8$, there is a dropdown of the temperature at $\theta=0$. At the beginning of the simulation, although the drop migrates from the cold region to the hot region as a whole, the temperature on the top of the drop still decreases due to the cold fluid rising from lower part of the drop. After $t=26$, the $\theta=0$ point will also become hotter because the system starts to go to equilibrium. On the other hand, the temperature at $\theta=\pi$ keeps increasing throughout the whole simulation because of the hot dropliquid from the top and hotter surrounding continuous phase. In general, the average difference of the temperature along the interface of the drop becomes smaller and smaller, and the temperature at the interface is almost a horizontal line at $t=26$. Because the interface tension $\sigma$ is a linear function of temperature, when the temperature gradient at the interface decreases, the drive force will reduce too. Figure 9 shows the time evolution of drop migration velocity, indicating an obvious increase-decrease process. Apparently, the overshoot is due to the decay of the drive force from $t=0$ to $t=26$. The migration velocity decreases around $t=8$, when the temperature at $\theta=\pi$ experiences a dramatic increase and the temperature at the drop interface is almost a horizontal line afterwards (Fig. 8).

There have been several previous reports on the overshoot phenomena of migration velocities. Oliver et al. calculated the transient thermocapillary motion of a gas bubble. ${ }^{27}$ They assumed that the gas bubble behaves as a constant volume void. Instead of solving the Navier-Stokes equations directly, the pseudo-steady-state velocities from Ref. 28 were adopted in their calculations. Although no physical explanation was given, there are obvious increase-decrease processes in the time evolutions of speeds in their paper. By solving the combination of Navier-Stokes equations and energy equation, a numerical investigation of the gas bubble was reported in Ref. 29. In the cases of bubble migrations, the effects of deformations are more important. ${ }^{19,20}$ However, from the discussion above, we know that the overshoot phenomena are mainly caused by the redistributions of temperature fields inside drops, and the influences of temperature redistributions in bubbles are much smaller because the densities of gases are much lower than those of liquids. As a result, the overshoot phenomena in their report are not as obvious as those in our simulations (e.g., see Figs. 8 and 9 in Ref. 29 and Fig. 9 in this paper). Also, similar phenomena have been reported in a numerical study of drops, ${ }^{17}$ which merely shows slight fluctuations because Ma numbers are smaller there.

However, in practice, it is difficult to have an initial con-

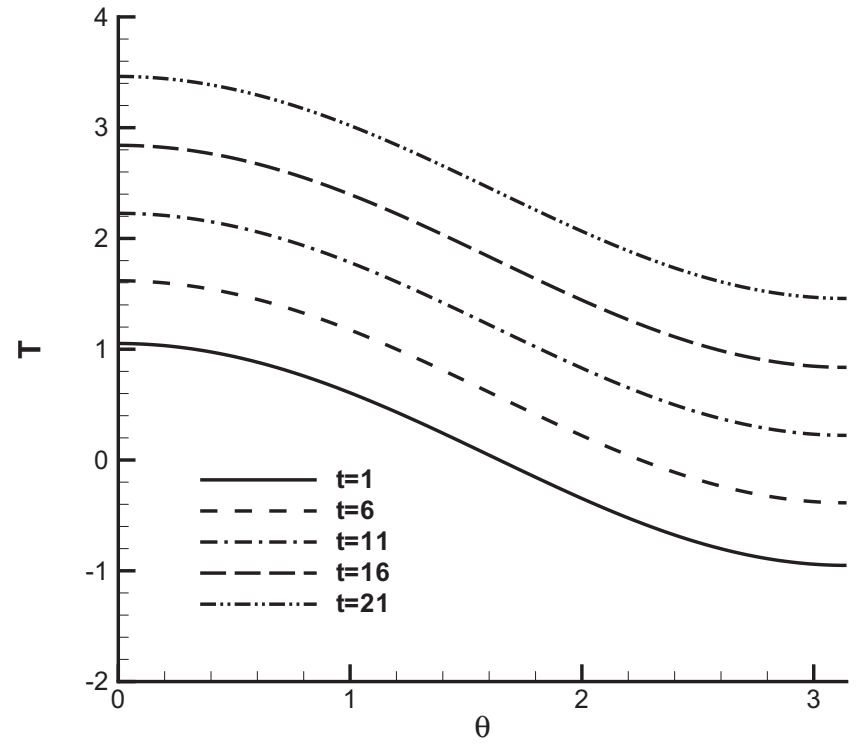

FIG. 12. Scaled temperature distribution along the drop interface for various time: $\mathrm{Ma}_{1}=1, \mathrm{Re}_{1}=1, \alpha=1$, and $\beta=1$. See the caption in Fig. 8 for the definition of $\theta$.

dition in experiments like what we adopt in this paper. In real experiments, drops are normally injected into continuous phases (see, e.g., Ref. 11). The initial velocities of drops cannot be exact zero. Moreover, no matter how short an injecting process is, the interface tension will inevitably take the time to drive the liquid inside the drop, and the temperature cannot be exactly linear along the $z$-direction, especially for large values of Ma. In this section and the following section, besides its easy implementation, we adopt such initial condition because of the tradition in related researches (see, e.g., the references in Sec. I). In Sec. V, we will extend our discussions to simulations starting from some other initial conditions, which are more reasonable from the view point of experiments.

\section{THE INFLUENCE OF NONDIMENSIONAL NUMBERS}

In this section, we will discuss what the above initial condition will lead to for different sets of the six most typical nondimensional numbers in the drop thermocapillary migration problem $\left(\mathrm{Ma}_{1}, \mathrm{Re}_{1}, \alpha, \beta, \xi\right.$, and $\left.\gamma\right)$. When we analyze the effect of one nondimensional number, the other five will be fixed to make the discussion easier. For each nondimensional number, when necessary, the situations for different values will be discussed.

It is also interesting to investigate how the final migration speeds are influenced by the nondimensional numbers. In this paper, most small values of $\mathrm{Ma}_{1}(<100)$ and a few large values of $\mathrm{Ma}_{1}$ runs are calculated until the final steady states are reached, but most runs of large values of $\mathrm{Ma}_{1}$ are terminated around $t=30$ to $t=100$, depending on the targeted nondimensional numbers.

To simplify our discussions, we let $\xi \equiv 1$ and $\gamma \equiv 1$ in the first two subsections (Secs. IV A and IV B). 


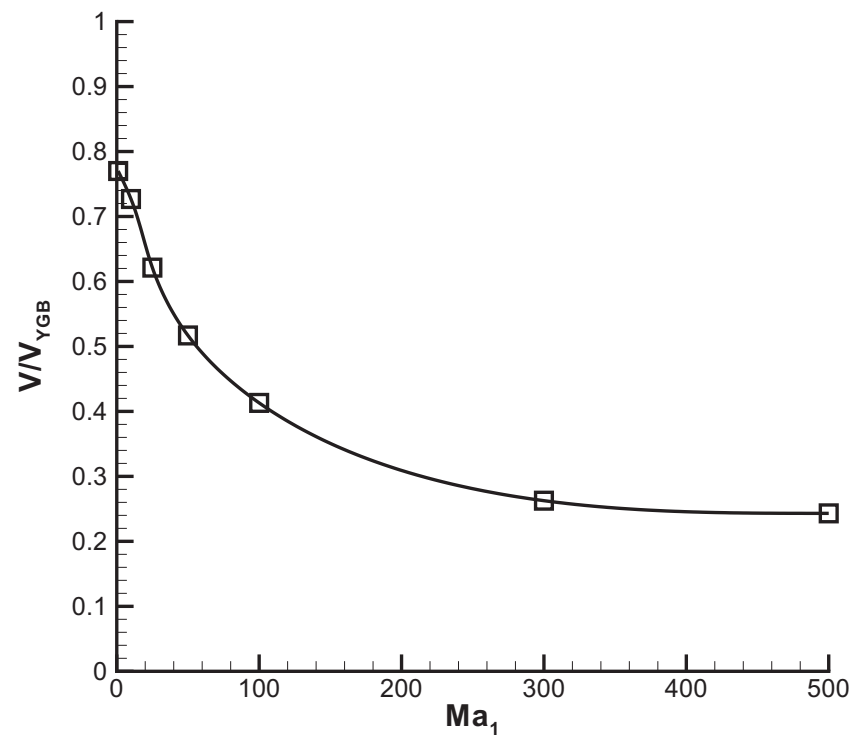

FIG. 13. The scaled drop migration velocity vs $\mathrm{Ma}_{1}, \operatorname{Re}_{1}=1, \alpha=1$, and $\beta=1$.

\section{A. The influence of Ma \\ 1. The influence of $\mathrm{Ma}_{1}$}

Figure 10 presents the time evolutions of drop migration velocity for different values of $\mathrm{Ma}_{1}$, and the rest values are fixed at $\operatorname{Re}_{1}=1, \alpha=1$, and $\beta=1$. For different values of $\mathrm{Ma}_{1}$, the initial migration velocities increase roughly at the same speed before $t \approx 2.0$. After $t \approx 2.0$, the migration velocity of the drop will reach a steady value directly when $\mathrm{Ma}_{1}$ is small (e.g., $\mathrm{Ma}_{1}=1$ ), without any oscillations in the process. However, there are obvious increasing-decreasing processes in the time-speed plots of large values of $\mathrm{Ma}_{1}$, and the decreasing degrees become larger for larger values of $\mathrm{Ma}_{1}$.

Figure 11 shows the temperature fields of different values of $\mathrm{Ma}_{1}$ at $t=28$, and the influence of $\mathrm{Ma}_{1}$ is much clearer.

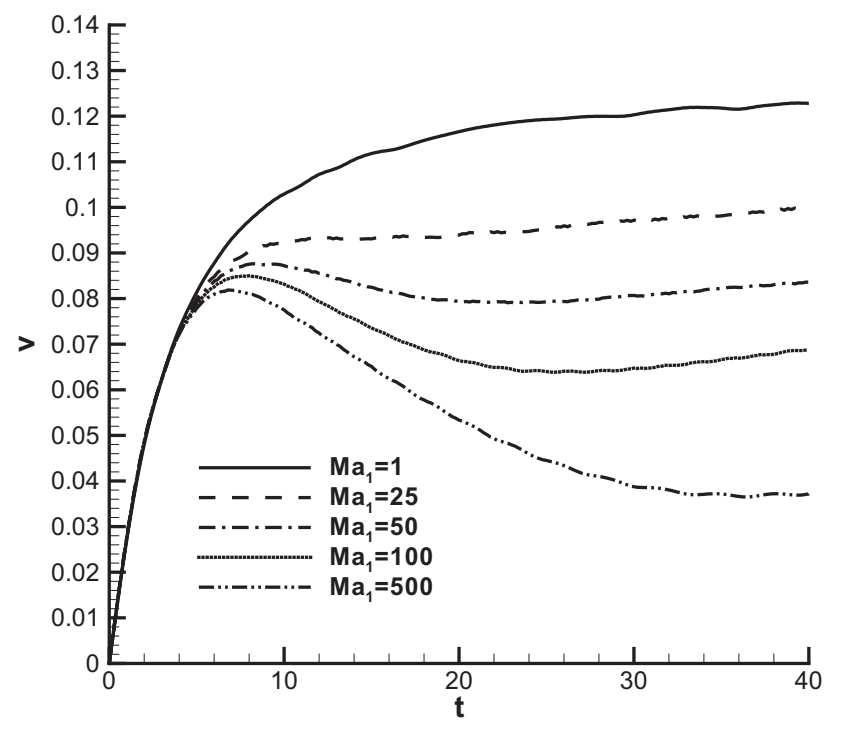

(a) $\operatorname{Re}_{1}=20$
The evolution of $\mathrm{Ma}_{1}=10$ is made into a movie [associated with Fig. 11(b)] to have a comparison with that of $\mathrm{Ma}_{1}$ $=500$ (the movie associated with Fig. 7).

There is almost no difference between the initial and the final temperature contour plots for small values of $\mathrm{Ma}_{1}$. The contour lines in Fig. 11(a) are almost straight just as those in the initial temperature field [Fig. 7(a)]. Throughout the $\mathrm{Ma}_{1}$ $=1$ simulation, the gradients of the temperature distribution along the interface are almost identical for the same $\theta$ (Fig. 12). In another word, while the drop is heated up by the surrounding fluid during the migration, $\nabla T(\theta)$ remains the same along the interface throughout the simulation and the surface-tension variance along the interface calculated with Eq. (13) also remains the same.

When $\mathrm{Ma}_{1}$ becomes larger, the contour lines of the temperature outside the top of the drop will wrap around the drop. Eventually, the final temperature along the interface is almost a constant for different values of $\theta$ (Fig. 8). Hence, in the case of a larger $\mathrm{Ma}_{1}$, the mechanism driving the drop becomes weaker shortly after the onset of the drop, which leads to a lower drop speed. Figure 13 shows the scaled drop migration velocities at $t=28$ versus $\mathrm{Ma}_{1}$ (scaled by $\left.V_{\mathrm{YGB}}\right)$. It is clear that the scaled migration velocities decrease with increasing values of $\mathrm{Ma}_{1}$.

Figure 14 gives the time evolutions of drop migration velocities for different values of $\mathrm{Ma}_{1}$ when the fixed values of $\mathrm{Re}_{1}$ are 20 and 40. The other two values are still fixed at $\alpha=1$ and $\beta=1$. Similar to Fig. 10, all large-Ma curves have increase-decrease processes in the early stages, and larger $\mathrm{Re}_{1}$ numbers lead to longer velocity-fluctuation periods. When $\mathrm{Ma}_{1}$ is big enough $\left(\mathrm{Ma}_{1}=500\right)$, or the heat convection is big enough compared to the heat diffusion, the migration velocity will experience an extra oscillation before reaching the steady state [Fig. 14(b)].

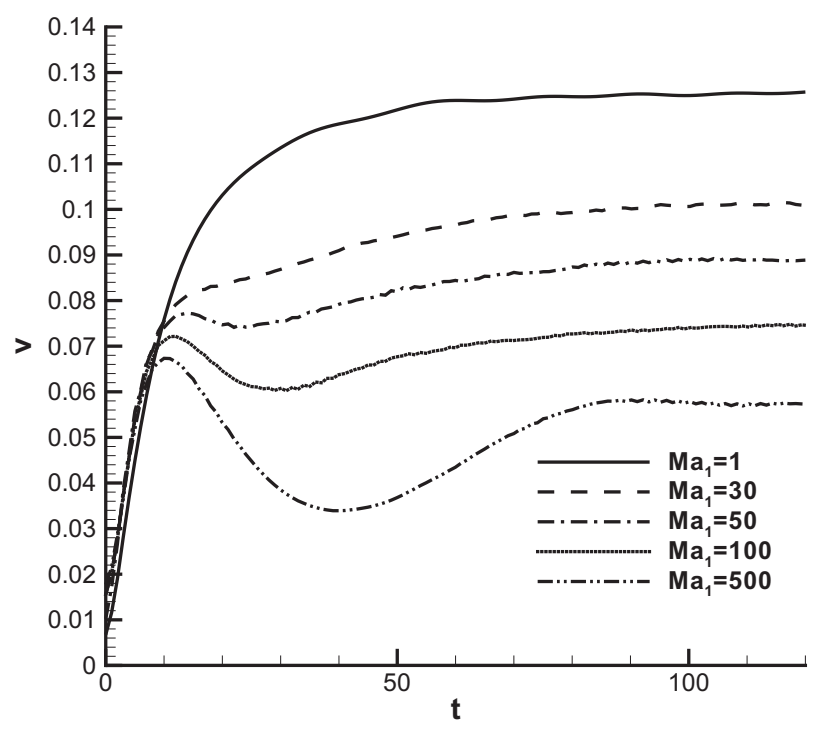

(b) $\operatorname{Re}_{1}=40$

FIG. 14. Time evolutions of drop migration velocity with different values of $\mathrm{Ma}_{1} . \alpha=1, \beta=1$, and $\operatorname{Re}_{1}=20,40$. 


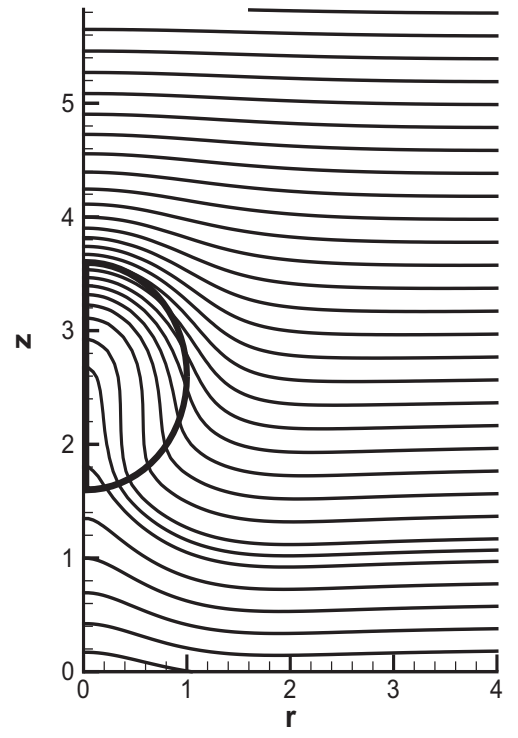

(a)

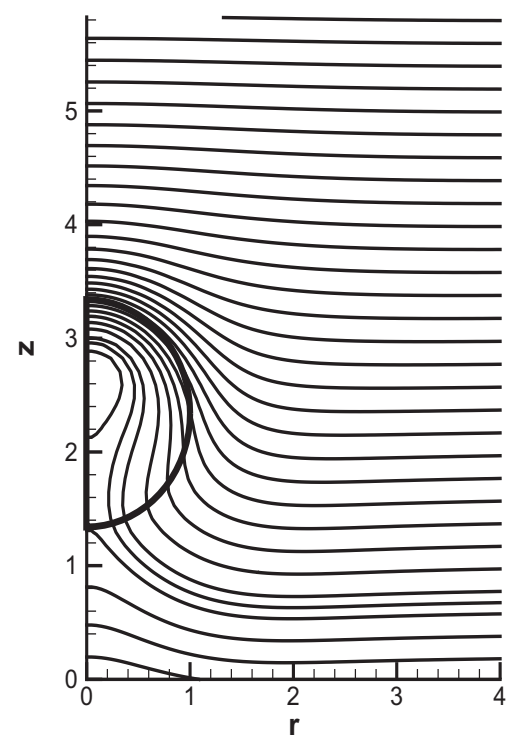

(b)

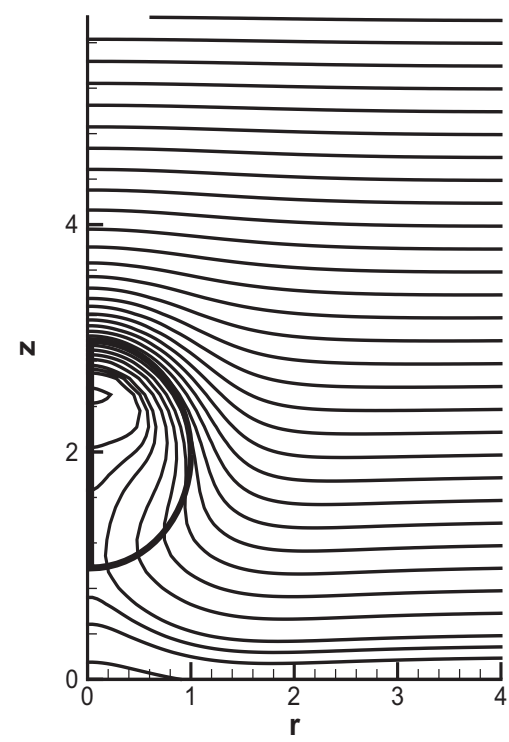

(c)

FIG. 15. The temperature field at $t=28, \mathrm{Ma}_{1}=100, \operatorname{Re}_{1}=1, \alpha=1$, (a) $\beta=2$, (b) $\beta=1$, and (c) $\beta=0.5$.

\section{The influence of $\beta$}

When the condition of the external fluid is fixed, the property ratio $\beta$ stands for the reciprocal of $\mathrm{Ma}_{2}$. Here, we only focus on the situation when $\mathrm{Ma}_{1}=100, \mathrm{Re}_{1}=1$, and $\alpha$ $=1$ to make the discussion concise. In Fig. 15, the temperature fields of three values of $\beta$ at $t=28$ are shown. For smaller thermal diffusivity ratio $(\beta)$ or larger $\mathrm{Ma}_{2}$ number, the heat convection inside the drop is stronger. In the case of $\beta=0.5$, the cold fluid has been pushed to the top of the drop before it is heated up [Fig. 15(c)], leading to a bigger reduction of the migration velocity. Unlike the temperature

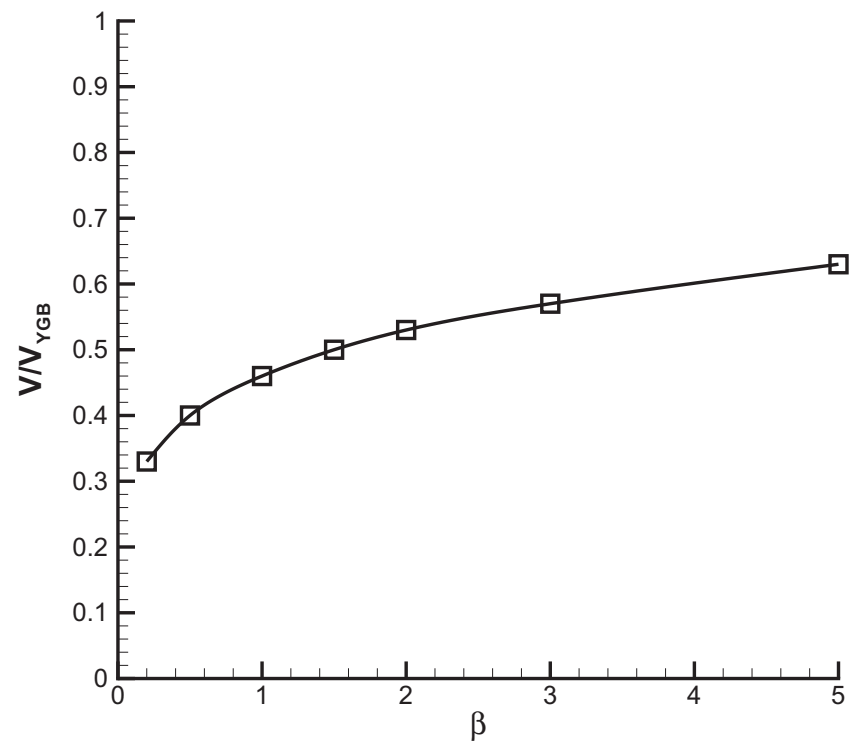

FIG. 16. The scaled drop migration velocity at $t=28$ vs thermal diffusivity ratios $(\beta), \mathrm{Ma}_{1}=100, \operatorname{Re}_{1}=1$, and $\alpha=1$. contours of the smaller values of $\beta$ at $t=28$, those of $\beta$ $=2.0[$ Fig. $15(\mathrm{a})]$ have not changed so dramatically compared to the initial ones [Fig. 7(a)] because the heat diffusion is the major mechanism, and the corresponding velocity is much larger since the capillary driving force keeps very strong throughout the simulation. The scaled migration velocities at $t=28$ versus thermal diffusivity ratios $(\beta)$ are shown in Fig. 16, and it is clear that the migration speed increases with the increasing $\beta$. In this sense, the role of the

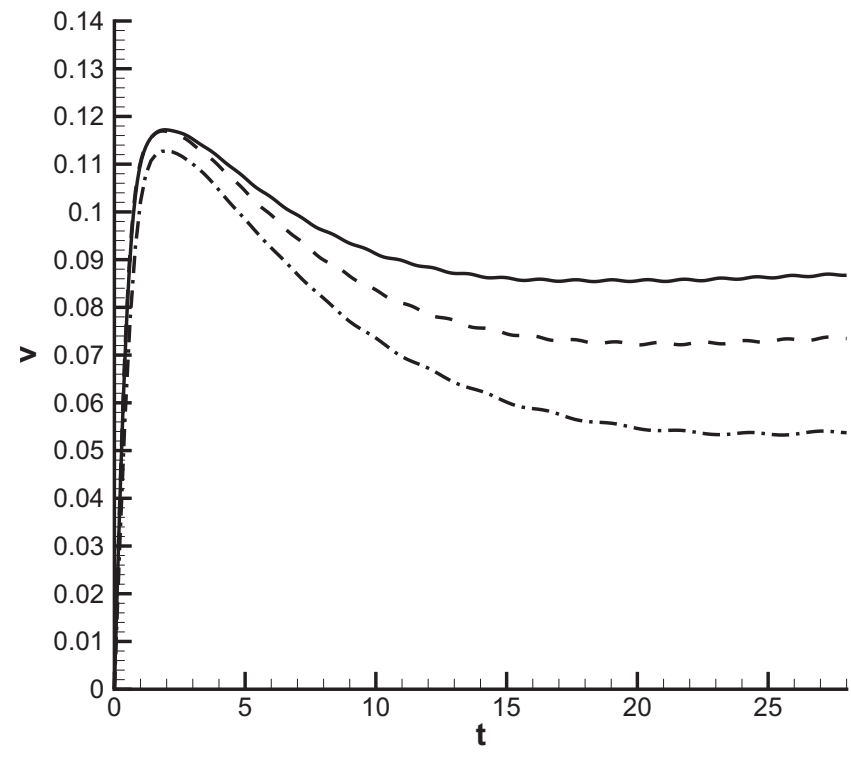

FIG. 17. Time evolutions of drop migration velocity with different thermal diffusivity ratios, $\beta=2(-), \beta=1(--), \beta=0.5(-\cdot-), \mathrm{Ma}_{1}=100, \mathrm{Re}_{1}=1$, and $\alpha=1$. 


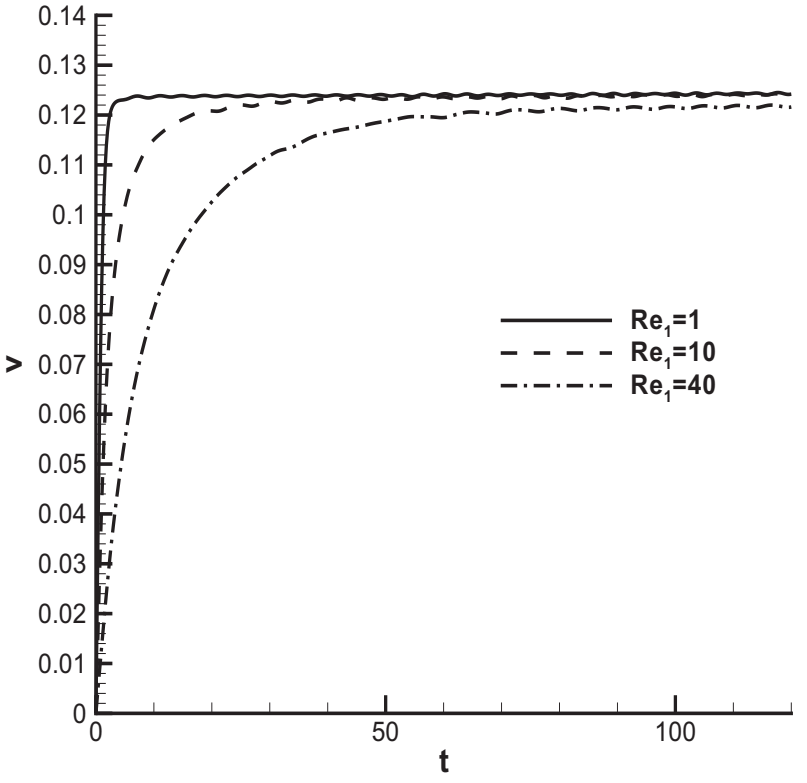

(a) $M a_{1}=1$

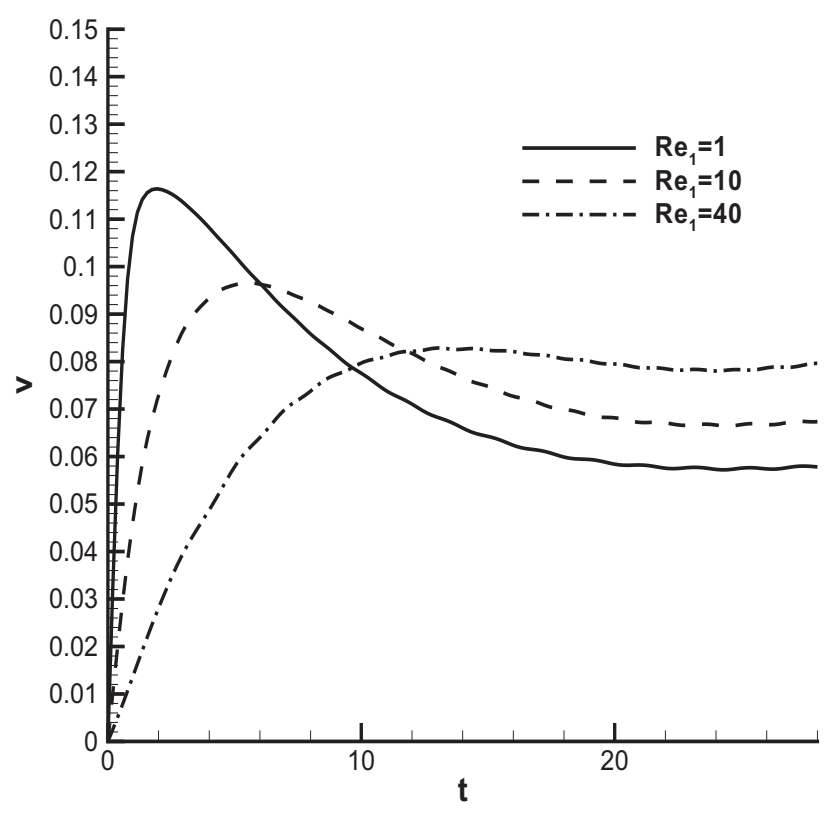

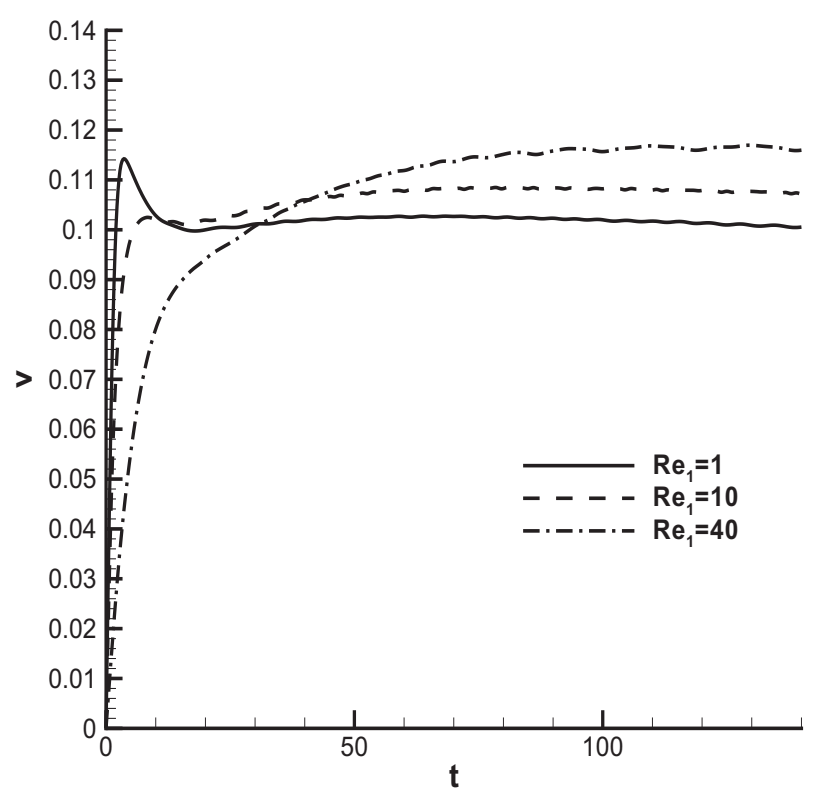

(b) $M a_{1}=20$

(c) $M a_{1}=100$

FIG. 18. Time evolutions of drop migration velocities with different values of $\operatorname{Re}_{1} \cdot \alpha=1, \beta=1$, and (a) $\mathrm{Ma}_{1}=1$, (b) $\mathrm{Ma}_{1}=20$, and (c) $\mathrm{Ma}_{1}=100$.

$\mathrm{Ma}_{2}($ or $1 / \beta)$ is the same as that of the $\mathrm{Ma}_{1}$ we have discussed in Sec. IV A 1.

The migration velocities with different thermal diffusivity ratios are shown in Fig. 17. Despite the different values of $\beta$, it always takes some time for cold liquid near the bottom of the drop to be transferred to the top. For this reason, the curves in Fig. 17 almost converge together in the beginning period. When the heat convection begins to take effect, the interface temperature of the drop is influenced by the fluid inside the drop, and the differences of the migration velocities for different values of $\beta$ appear.

\section{B. The influence of Re 1. The influence of $\mathrm{Re}_{1}$}

In the former studies of the drop migration problem, the role of $\mathrm{Re}_{1}$ has not drawn too much attention because the variance of $\operatorname{Re}_{1}$ has much smaller influence on the final steady migration velocity than that of $\mathrm{Ma}_{1}$ does. In the following, however, we will show that the unsteady migration processes of drops are very different for varying values of $\mathrm{Re}_{1}$, especially in the cases of large values of $\mathrm{Ma}_{1}$. 


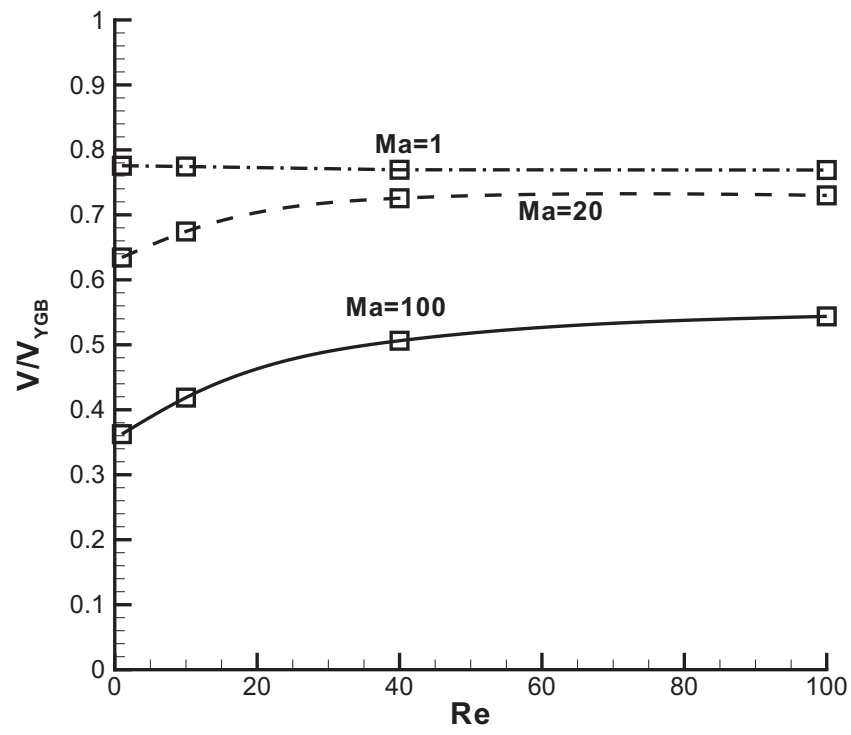

FIG. 19. The scaled drop migration velocity vs $\mathrm{Re}_{1}, \mathrm{Ma}_{1}=1,20$, and 100 . The values of the velocities related to the $M a=100$ runs are from the states at $t=28$ instead of the final states.

The major difficulty in the simulations of this subsubsection is to remove the influence of the reference velocity. The following is a comparison of the definition of $\operatorname{Re}_{1}$ and $U$ :

$$
\begin{aligned}
& \operatorname{Re}_{1}=\frac{R U}{\nu_{1}}=\frac{\rho_{1} R U}{\mu_{1}}, \\
& U=\frac{\left|\sigma_{T}\right| \nabla T R}{\mu_{1}} .
\end{aligned}
$$

Consequently, to make the reference velocities in this subsubsection unique within each figure, we have to change the values of $\nabla T$ in the meanwhile of changing $\operatorname{Re}_{1}$ so the value of $\nabla T / \mu_{1}$ is fixed.

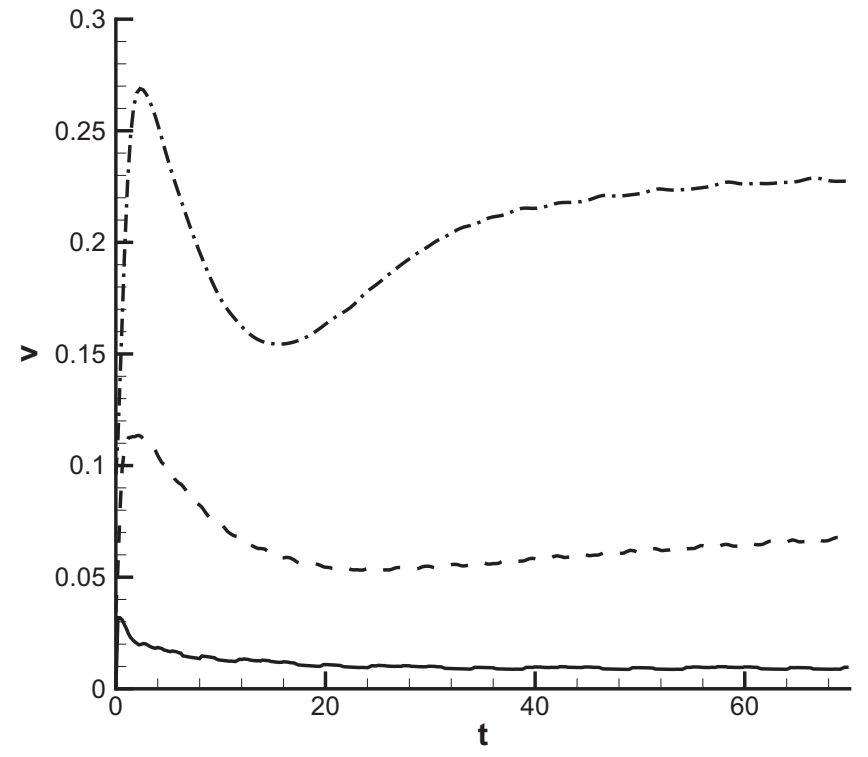

FIG. 21. Time evolutions of drop migration velocities with different kinematic viscosity ratios: $\alpha=2(-), \alpha=1(--), \alpha=0.5(-\cdot-) ; \mathrm{Ma}_{1}=100$, $\operatorname{Re}_{1}=1, \beta=1$.

Figure 18 show the time evolutions of drop velocities under different values of $\mathrm{Re}_{1}$. The most obvious difference between Fig. 18 and the corresponding pictures in the previous subsection (Figs. 10, 14, and 17) happens at the beginning part of the simulations. Different values of $\operatorname{Re}_{1}$ show nontrivial influence on the beginning part of the simulations. On the other hand, although simulations of different values of $\mathrm{Ma}_{1}$ or $\beta$ have quite different drop velocities at the later stages, the initial migration processes of them are close to each other.

To have a better understanding of the impact of $\mathrm{Re}_{1}$, it is necessary to understand the mechanism of the thermocapillary migration. The force driving the drop is actually the

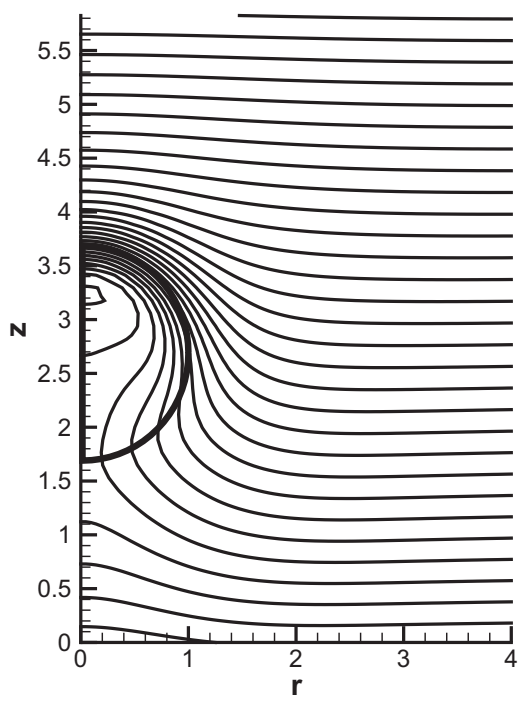

(a)

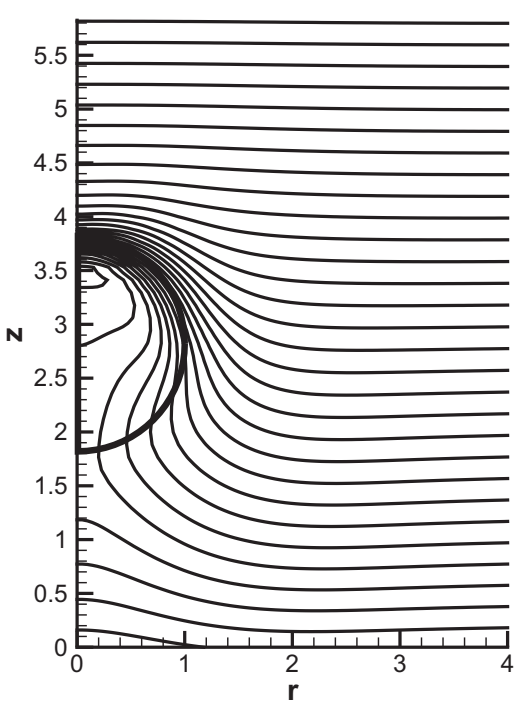

(b)

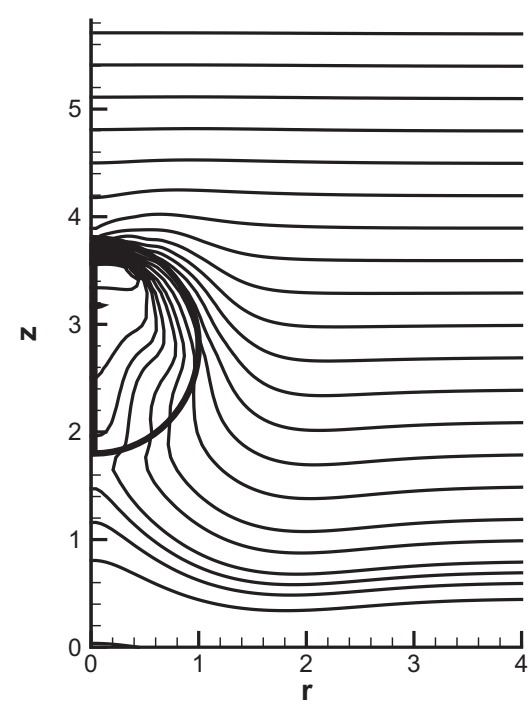

(c)

FIG. 20. The temperature field at $t=28, M_{1}=100, \alpha=1, \beta=1$; and (a) $\operatorname{Re}_{1}=1$, (b) $\operatorname{Re}_{1}=10$, (c) $\operatorname{Re}_{1}=40$. 


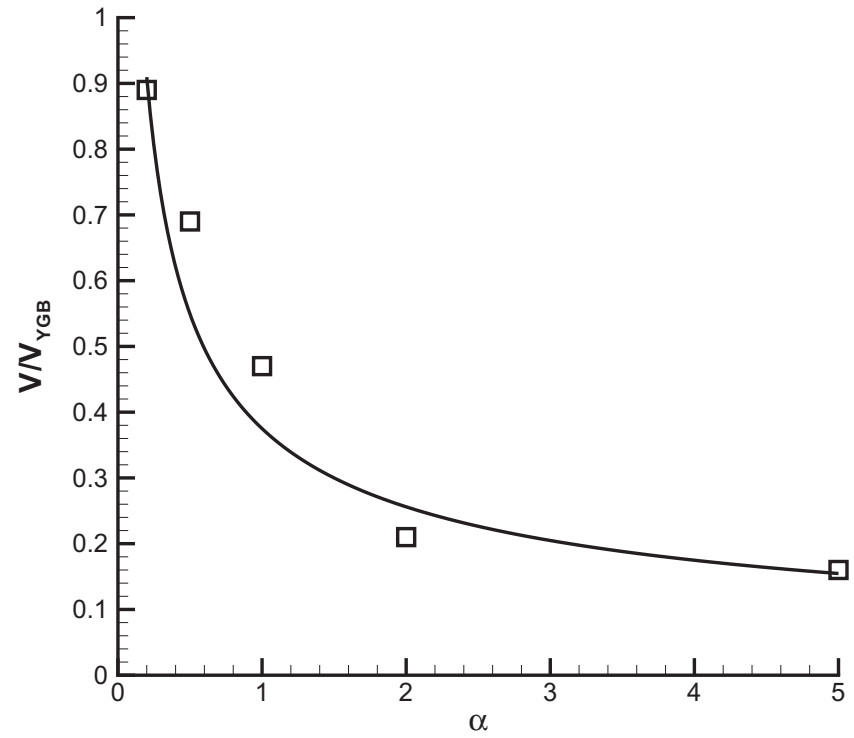

FIG. 22. The scaled migration velocities vs $(\alpha)$ at $t=70, M_{1}=100, \operatorname{Re}_{1}$ $=1$, and $\beta=1$. The line indicates the least squares fitting of the numerical data.

counterforce when the interface tension drives the background liquid near the interface through the effect of viscosity. The viscosity force of the background liquid can be viewed as the driving force and the resistance of the drop migration simultaneously. From Fig. 18, it is clear that the simulation with the smaller $\operatorname{Re}_{1}$ tends to move slower at the late stage of the simulation because the resistance is larger \{the three $\mathrm{Ma}_{1}=1$ runs are exceptions [Fig. 18(a)], but actually the final parts of their speed curves are very close to each other\}. On the other hand, at the beginning of the simulations, there is no difference between the interface tensions of different values of $\operatorname{Re}_{1}$ because their initial temperature distributions are the same. Smaller values of $\operatorname{Re}_{1}$ mean larger viscosities and drops will have larger acceleration in the beginning. Hence, compared to larger values of $\mathrm{Re}_{1}$, smaller $\mathrm{Re}_{1}$ drops can reach their maximum velocities within shorter periods.

When $\mathrm{Ma}_{1}$ is small [Fig. 18(a)], there is no fluctuation before migration velocity reaches the equilibrium state. The temperature contours of the steady states of different $\mathrm{Re}_{1}$ runs show similar patterns; therefore, if $\mathrm{Ma}_{1}$ is small, $\mathrm{Re}_{1}$ 's effect on the final velocity of the drop is also rather small. For example, when $\mathrm{Ma}_{1}=1$ the steady migration velocities are almost identical for different values of $\operatorname{Re}_{1}$ [Fig. 18(a)], and the only difference is the migration time to reach the steady state.

In the case of $\mathrm{Ma}_{1}=20$ [Fig. 18(b)], the difference of steady migration velocities for different values of $\mathrm{Re}_{1}$ appears, and larger values of $\mathrm{Re}_{1}$ also need more time to reach the steady states. The variation of the scaled drop migration velocity for different values of $\operatorname{Re}_{1}$ is shown in Fig. 19. It is clear that the scaled migration velocities slightly increase with increasing values of $\mathrm{Re}_{1}$, which is similar to the results in Ref. 16, and these increasing rates of final speeds are higher for larger values of $\mathrm{Ma}_{1}$. The $\mathrm{Ma}_{1}=100$ runs have not been calculated long enough to reach their final states, but we can roughly draw the similar conclusions.

Generally speaking, the temperature contours of different values of $\mathrm{Re}_{1}$ at the late stages are quite close to each other. Subtle difference in temperature field can be found near the top of the drop when $\mathrm{Ma}_{1}$ is fairly large (Fig. 20). The cold region inside the drop is closer to the top of the drop for larger $\mathrm{Re}_{1}$, and the continuous phase just above the drop is also affected, leading to the bending down of the isothermal lines [Fig. 20(c)].

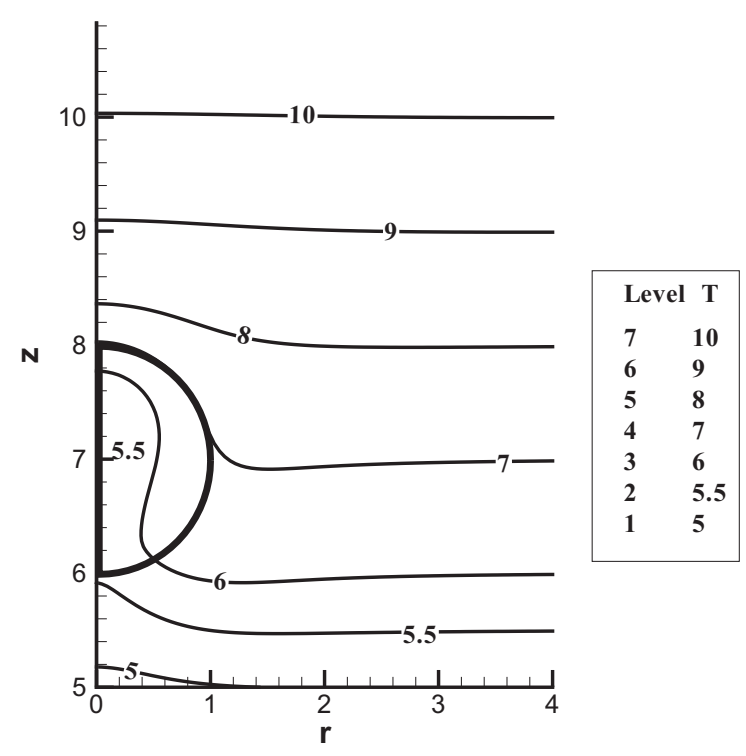

(a) $t=10$

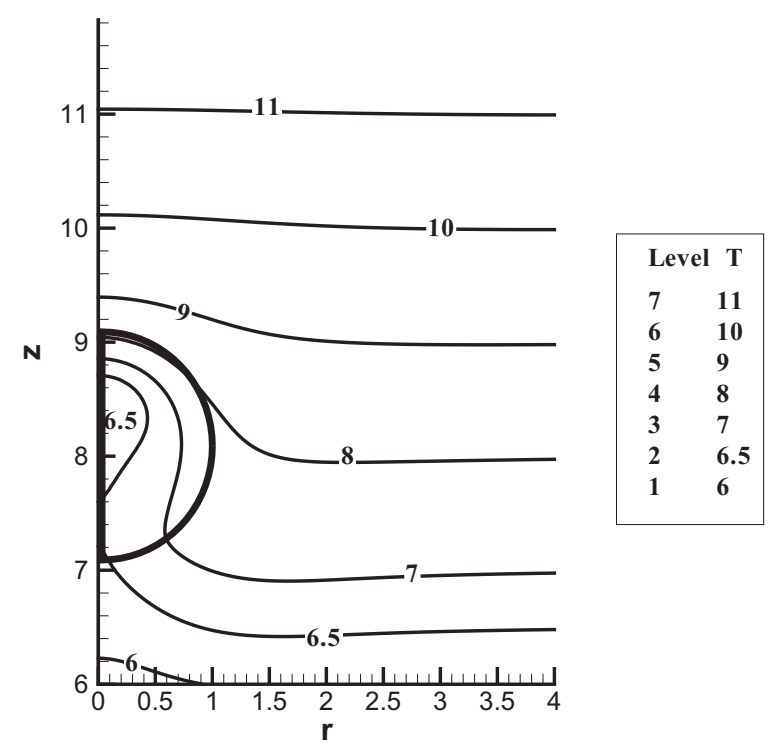

(b) $t=20$

FIG. 23. The contour plots of temperature for simulations with $\xi=0.5\left(\operatorname{Re}_{1}=1, \mathrm{Ma}_{1}=100, \alpha=1, \beta=1\right.$, and $\left.\gamma=1\right)$. 


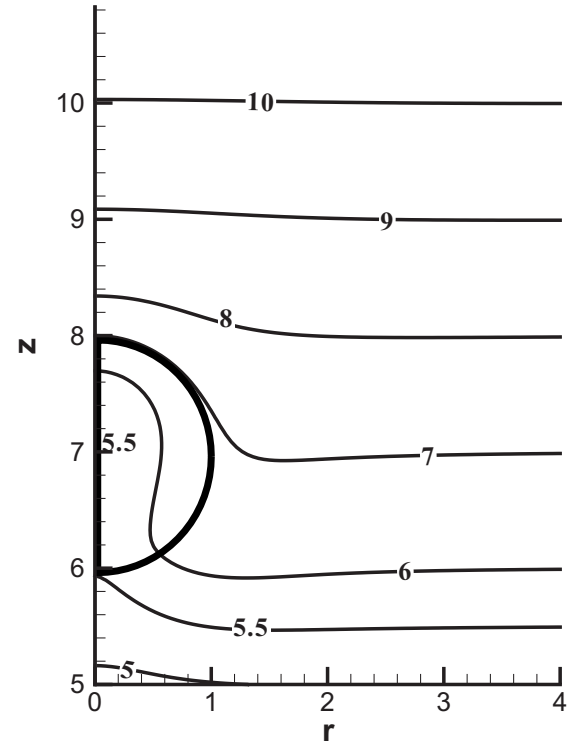

(a) $t=10$

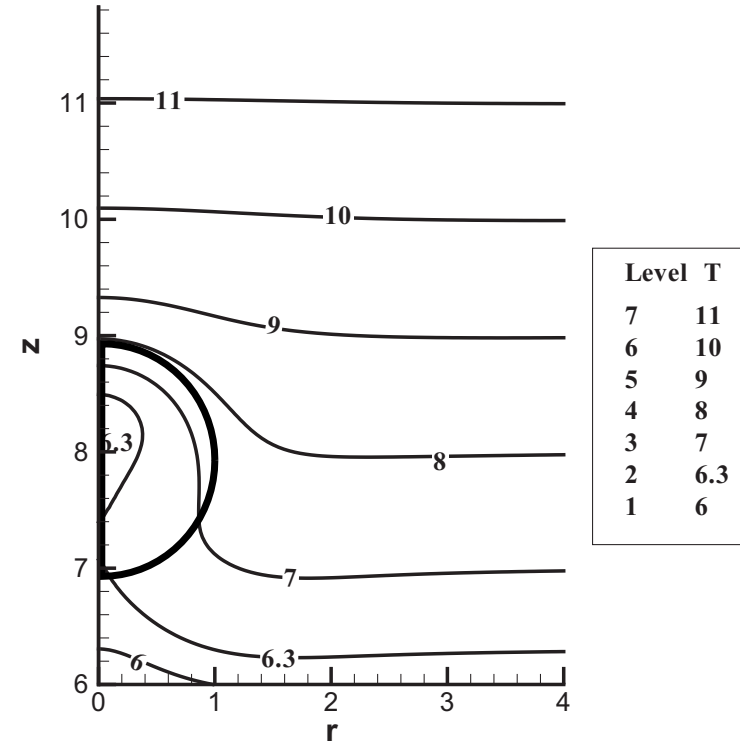

(b) $t=20$

FIG. 24. The contour plots of temperature for simulations with $\xi=1\left(\operatorname{Re}_{1}=1, \mathrm{Ma}_{1}=100, \alpha=1, \beta=1\right.$, and $\left.\gamma=1\right)$.

\section{The influence of $\alpha$}

When the condition of the external fluid is fixed, the property ratio $\alpha$ stands for the reciprocal of $\operatorname{Re}_{2}$. From the discussion on the role of $\mathrm{Re}_{1}$, we find that Reynolds numbers can greatly affect the migration evolution of the drop when $\mathrm{Ma}_{1}$ is large; therefore, in this subsubsection, the $\mathrm{Ma}_{1}$ is fixed at 100 to have a brief view of the properties of $\alpha$.

Figure 21 shows the velocity evolutions for three values of $\alpha$. It seems that comparing with other five nondimensional numbers $\left(\mathrm{Ma}_{1}, \mathrm{Re}_{1}, \beta, \xi\right.$, and $\left.\gamma\right), \alpha$ has the largest influence on the migration history of the drop.

From the definition of $\alpha$ [Eq. (4)], it is known that larger values of $\alpha$ mean larger viscosities inside drops. Larger viscosities mean weaker fluid convections. Smaller velocities inside more viscous drops lead to lower speeds outside the drops because of the continuity of the velocities near the interfaces; as a result, more viscous drops have smaller migration velocities.

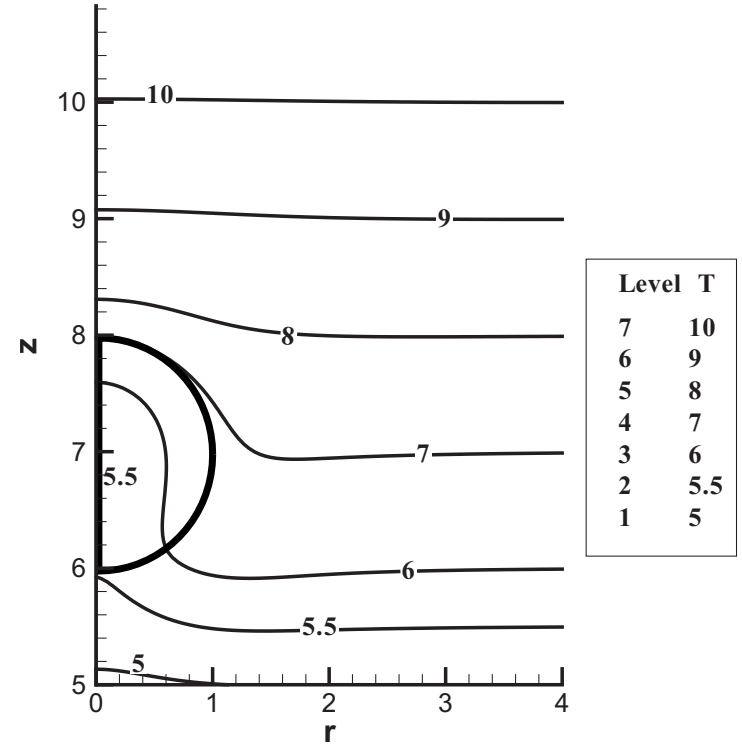

(a) $t=10$

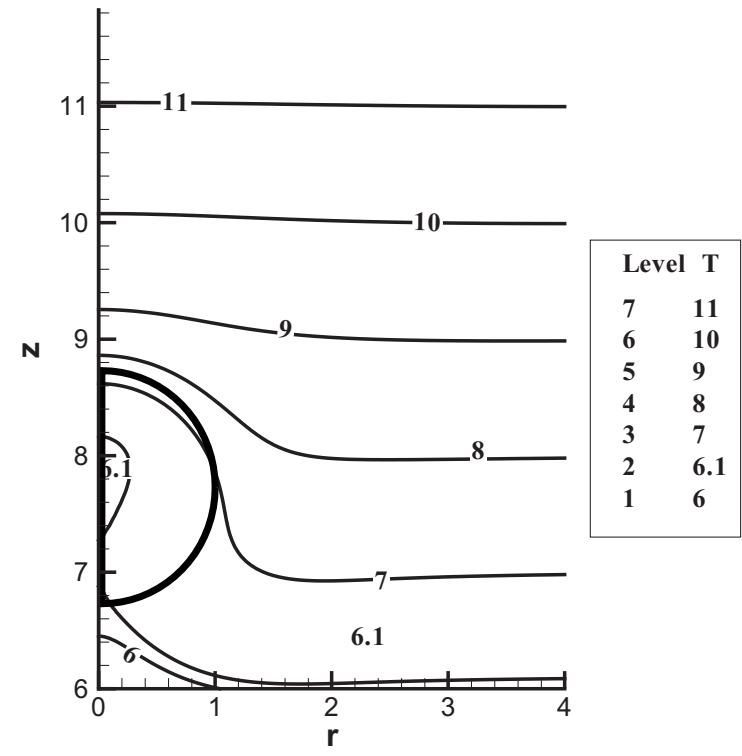

(b) $t=20$

FIG. 25. The contour plots of temperature for simulations with $\xi=2\left(\operatorname{Re}_{1}=1, \mathrm{Ma}_{1}=100, \alpha=1, \beta=1\right.$, and $\left.\gamma=1\right)$. 


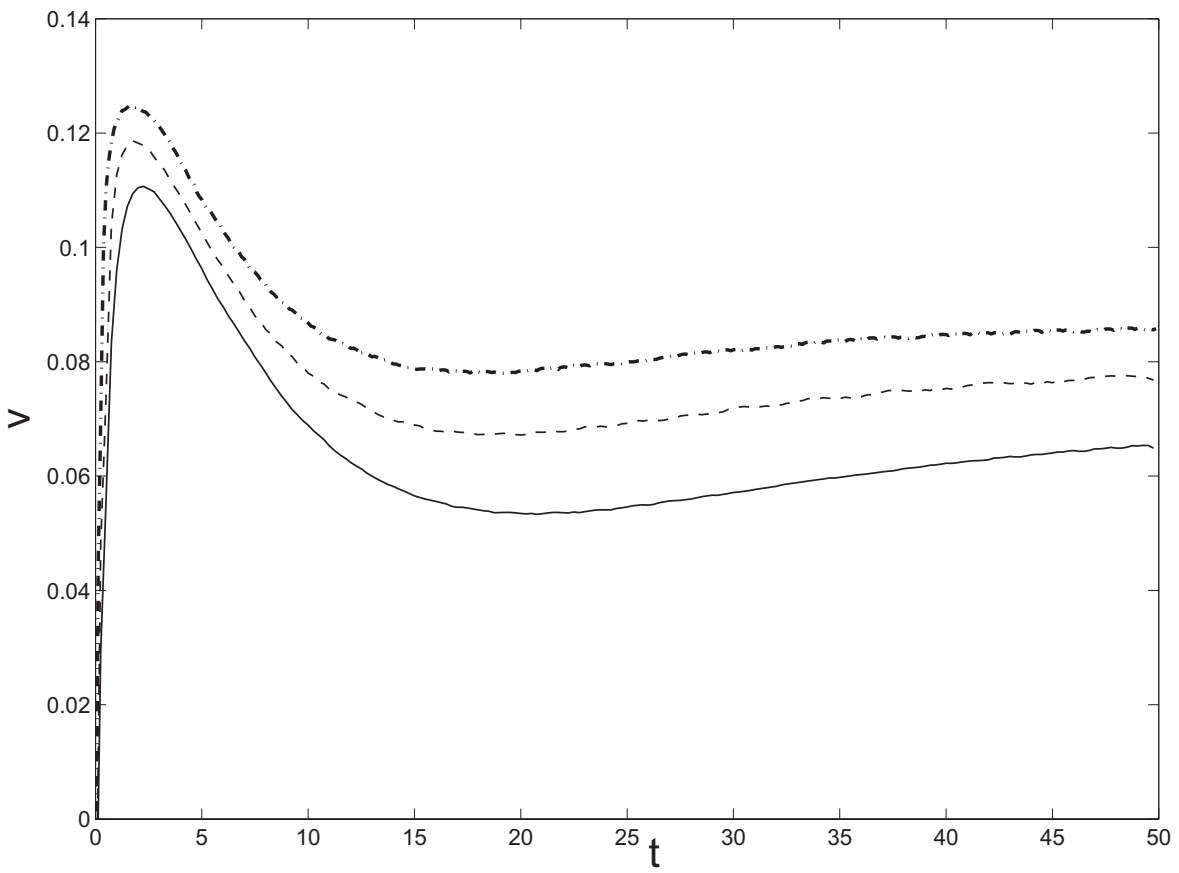

FIG. 26. The time evolutions of migration velocities for three runs in different drop densities. $\mathrm{Re}_{1}=1, \mathrm{Ma}_{1}$ $=100, \alpha=1, \beta=1$, and $\gamma=1$ (dashdotted line: $\xi=0.5$; dashed line: $\xi=1$; solid line: $\xi=2$ ).

(a) $\gamma=0.25$

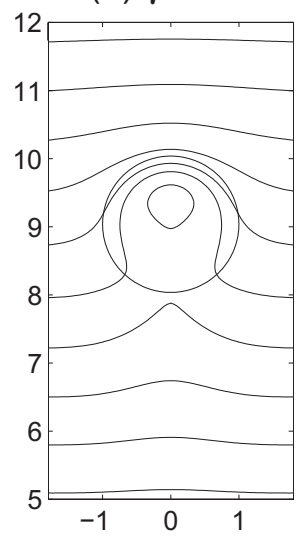

(b) $\gamma=0.50$

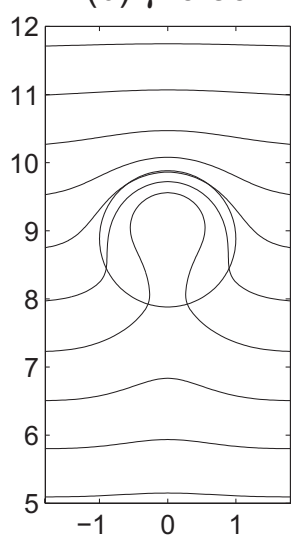

(c) $\gamma=1$

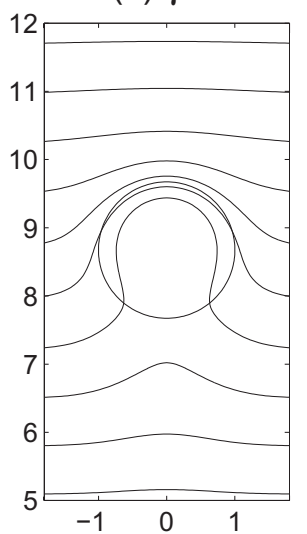

(d) $\gamma=2.0$

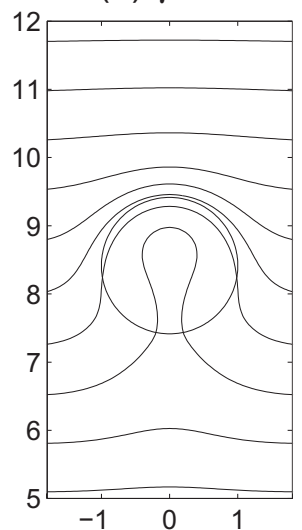

(e) $\gamma=4.0$

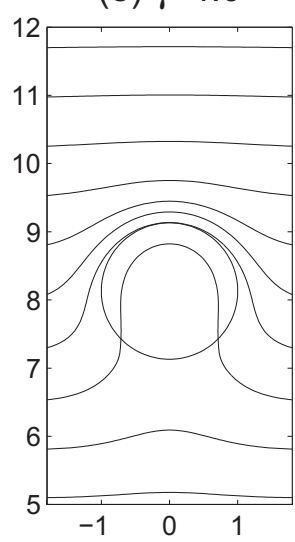

FIG. 27. The contour plots of temperature for simulations with different values of $\gamma$ at $t=29.0\left(\operatorname{Re}_{1}=1, \operatorname{Ma}_{1}=100, \alpha=1, \beta=1\right.$, and $\left.\xi=1\right)$.

(a) $\gamma=0.25$

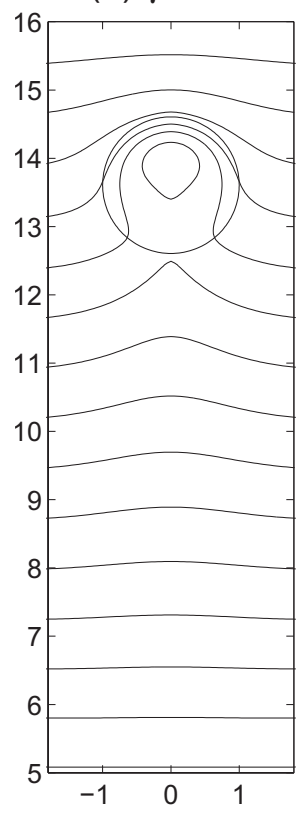

(b) $\gamma=0.50$

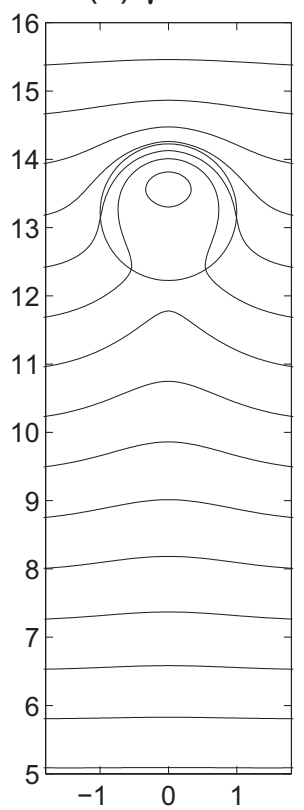

(c) $\gamma=1$

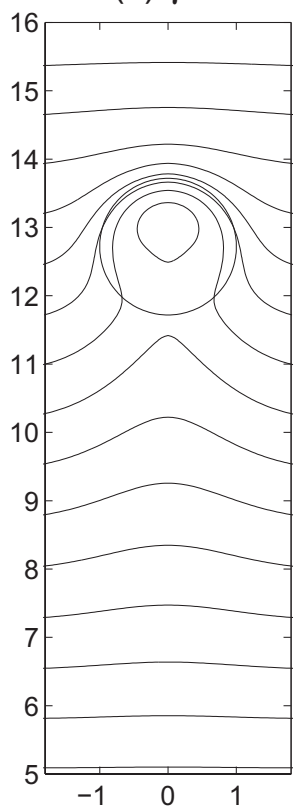

(d) $\gamma=2.0$

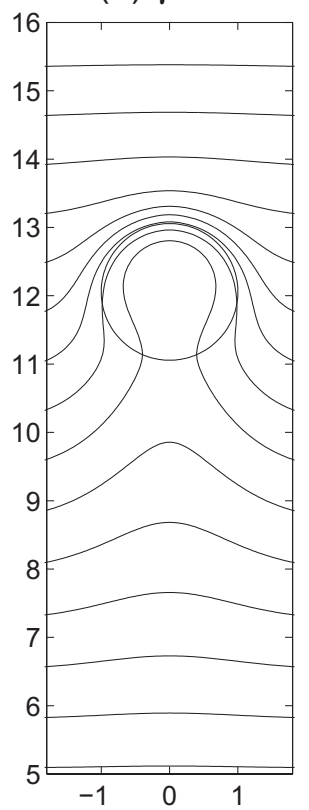

(e) $\gamma=4.0$

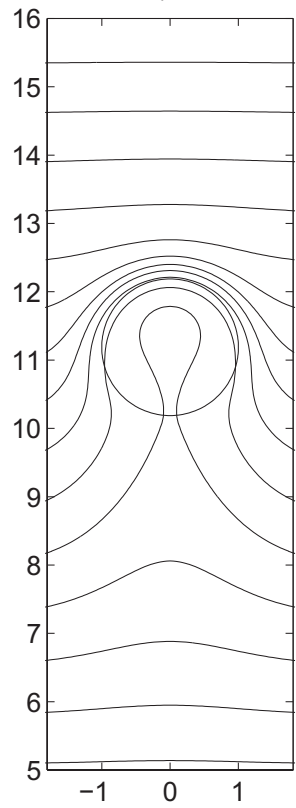

FIG. 28. The contour plots of temperature for simulations with different values of $\gamma$ at $t=74.0\left(\operatorname{Re}_{1}=1, \operatorname{Ma}_{1}=100, \alpha=1, \beta=1\right.$, and $\left.\xi=1\right)$. 


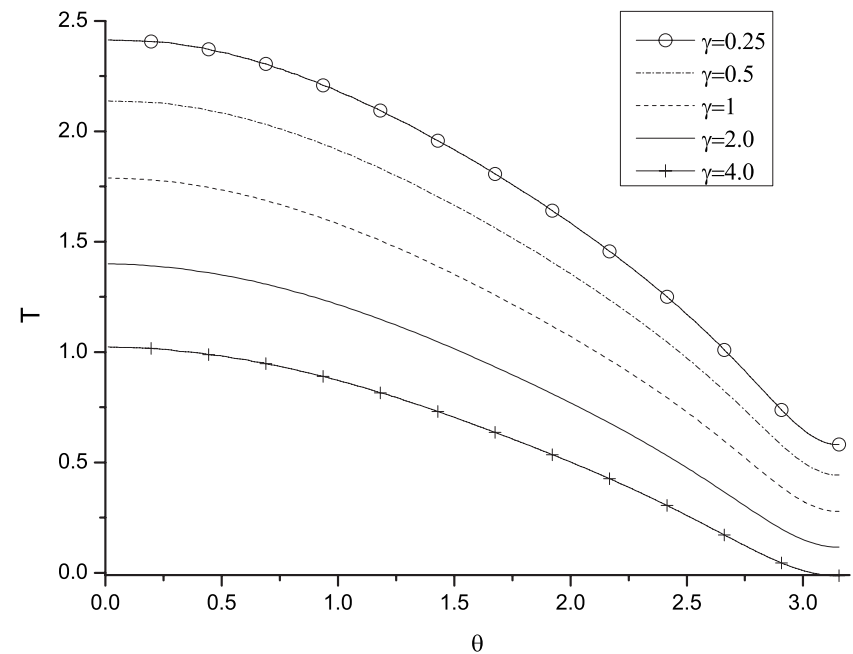

FIG. 29. Scaled temperature distributions along drop interfaces for different drop specific heats at $t=20 . \operatorname{Re}_{1}=1, \mathrm{Ma}_{1}=100, \alpha=1, \beta=1$, and $\xi=1$. See the caption in Fig. 8 for the definition of $\theta$.

When $\alpha$ is small, the smaller viscosity leads to stronger vortices inside the drop, there is more obvious undulation in the migration velocity due to the stronger heat convection effect (Fig. 21). In the case of $\alpha=0.5$, the velocity experiences an up-down-up process at the beginning part of the simulation.

The plot of scaled migration velocities versus kinematic viscosity ratios $(\alpha)$ at $t=70$ is given in Fig. 22 . The migration velocity indicates obvious decrease when $\alpha$ increases.

\section{The influence of $\xi$}

In experiments, it is difficult to have enough microgravity time in the drop tower, and it is very expensive to perform experiments in the space. The cheapest way to get long-time microgravity condition in experiments is the so-called density-match methods, which choose pairs of drops and background liquids with equal densities. ${ }^{7-9}$ For this reason, it is quite necessary to study the situation when the value of $\xi$ is fixed to 1 .

However, the role of $\xi$ in thermocapillary migrations is also very interesting. The biggest difference between the drop and the bubble is the different ratios of the drop/bubble density to that of the background fluid. The bubble means that $\xi$ is in the order of $10^{-2}$ or $10^{-3}$, while the drop means $\xi$ is $O(1)$. It is apparent that the flow inside the drop will also influence the drop migration, and for this reason the phenomena associated with drops may be more complex than those of bubbles. It is impossible to discuss all situations corresponding to all possible values of $\xi$ while having the variances of $\mathrm{Re}_{1}, \mathrm{Ma}_{1}, \alpha, \beta$, and $\gamma$ considered in one paper. In the following, we will only make the value of $\xi$ vary around 1 , and all other nondimensional parameters remain the same: $\operatorname{Re}_{1}=1, \mathrm{Ma}_{1}=100, \alpha=1, \beta=1$, and $\gamma=1$.

It is predictable that the lighter drop will move faster when the status outside of drops is fixed. On the other hand, migration velocities are also determined by temperature distributions along the interface. The influence of $\xi$ is much more obvious if we compare the isothermal lines at $t=10$ of the three runs. We will concentrate on the contour lines with $T=7$ (or level 4 described in the legends) of the three temperature contour plots. In the case of $\xi=0.5$, the $T=7$ line wraps almost half of the interface [Fig. 23(a)]. The overlap part between the interface and the $T=7$ line becomes obviously smaller when $\xi$ becomes larger $[\xi=1$ : Fig. $24(\mathrm{a}) ; \xi$ =2: Fig. 25(a)], indicating larger temperature variance along the interface. Therefore, although the lightness of a drop can reduce its inertia, it can also make the redistribution of temperature inside the drop easier, leading to smaller driving

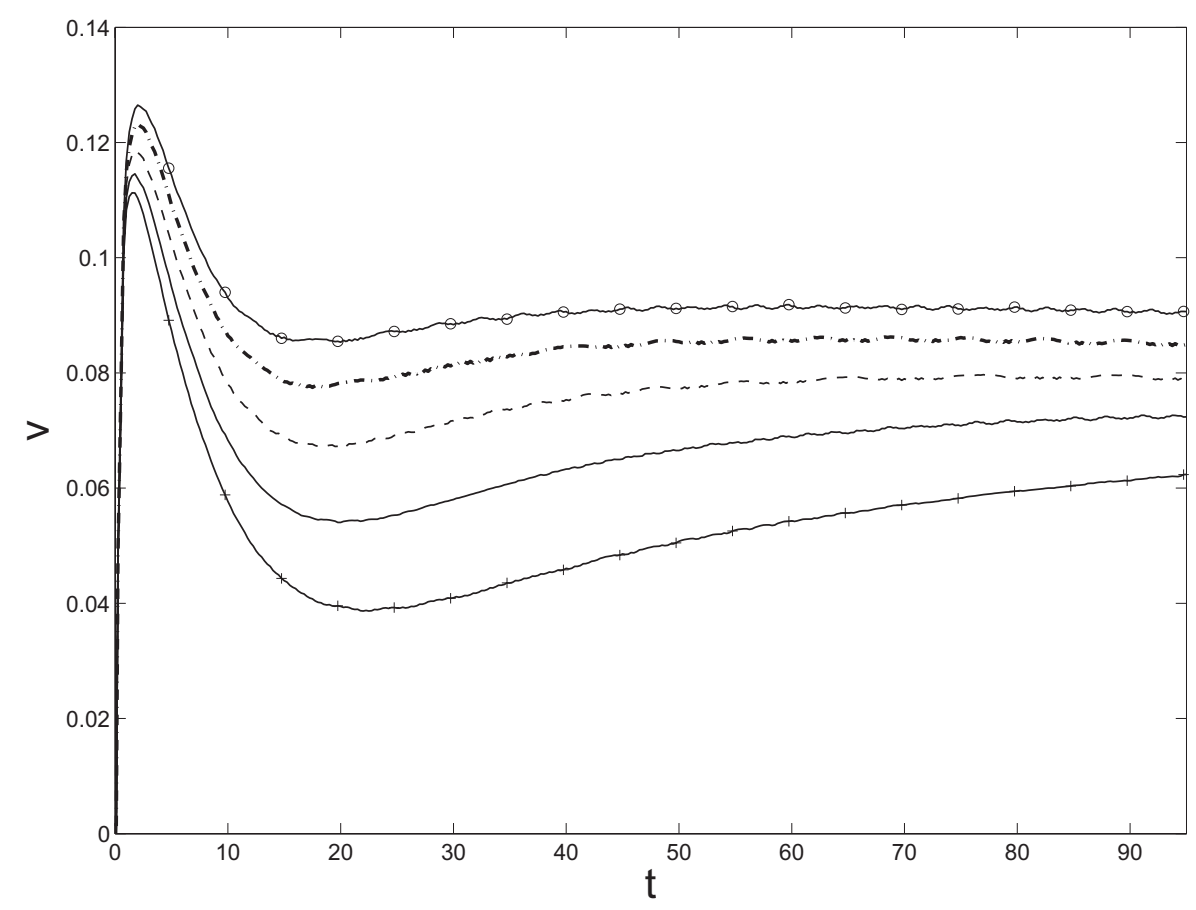

FIG. 30. The time evolutions of migration velocities for five runs with different drop specific heats. $\operatorname{Re}_{1}=1$, $\mathrm{Ma}_{1}=100, \alpha=1, \beta=1$, and $\xi=1:(1)$ solid-circle line: $\gamma=0.25$; (2) dashdotted line: $\gamma=0.5$; (3) dashed line: $\gamma$ $=1$; (4) solid line: $\gamma=2.0$; (5) solidcross line: $\gamma=4.0$. 


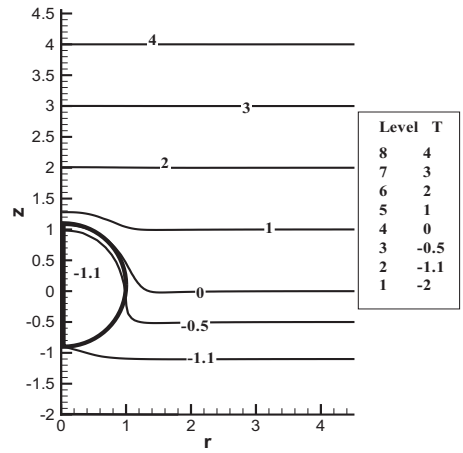

(a) $\mathrm{t}=2$

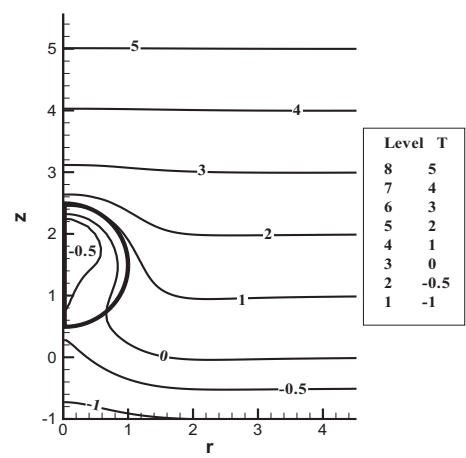

(b) $\mathrm{t}=20$

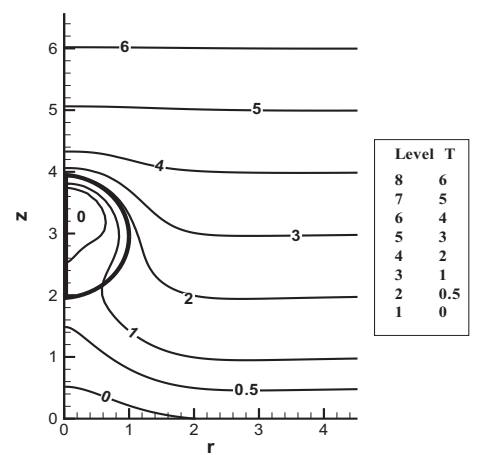

(c) $\mathrm{t}=40$

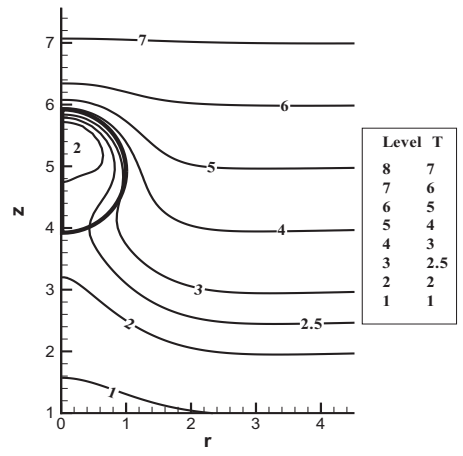

(d) $\mathrm{t}=66$

FIG. 31. The isotherm plots of Run A

force on the drop. For this reason, the doubling of the drop density does not lead to the halving of the migration speed. As indicated in Fig. 26, the speed of the $\xi=0.5$ run at $t=50$ is only $11.5 \%$ faster than that of $\xi=1$, and the speed of the $\xi$ $=1$ run at $t=50$ is only $21.6 \%$ faster than that of $\xi=2$.

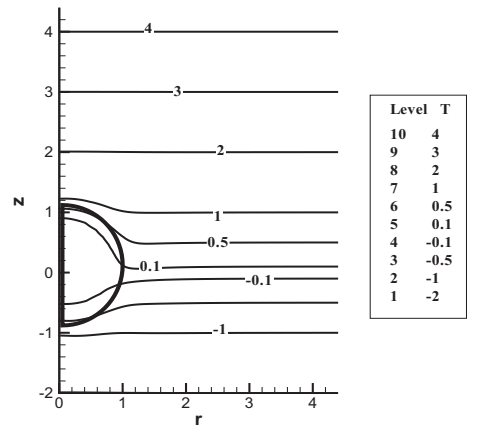

(a) $t=2$

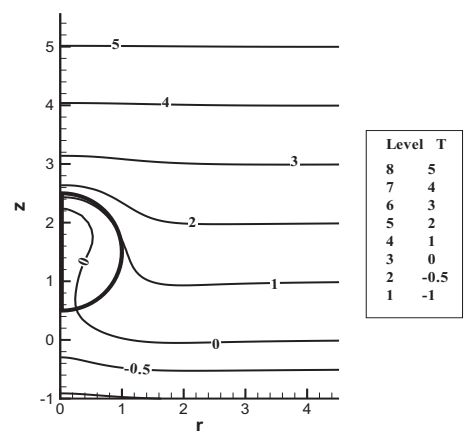

(b) $t=20$

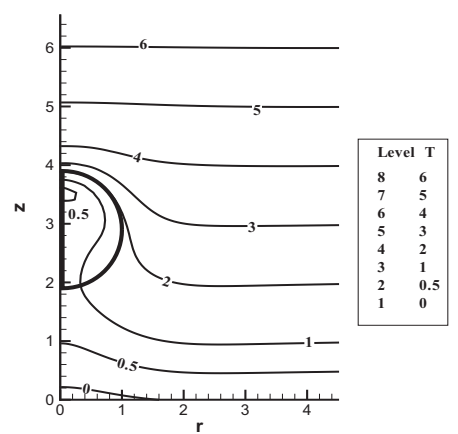

(c) $\mathrm{t}=40$

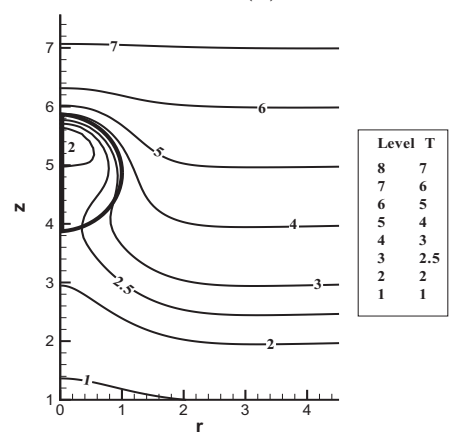

(d) $\mathrm{t}=66$

FIG. 32. The isotherm plots of Run B.

\section{The influence of $\gamma$}

In the experiments of thermocapillary migration, the specific heats of both liquids are normally very close to each other. For example, in the widely adopted vegetable oil and silicone oil experimental system, the specific heats of two liquids are very close (vegetable oils vary from 1 to $1.38 \mathrm{~kJ} / \mathrm{K} \mathrm{g}{ }^{\circ} \mathrm{C}$; DOW-Corning DC200 5cs: $C_{p}$ $\left.=1.34 \mathrm{~kJ} / \mathrm{K} \mathrm{g}{ }^{\circ} \mathrm{C}\right)$. This is the reason why we assume $\gamma \equiv 1$ 
in the other parts of the paper. On the other hand, the role of $\gamma$ is also very interesting. We will make the value of $\gamma$ vary around 1, and all other nondimensional parameters remain the same: $\operatorname{Re}_{1}=1, \mathrm{Ma}_{1}=100, \alpha=1, \beta=1$, and $\xi=1$.

The major difference on the temperature contour plots with different values of $\gamma$ can be found in the contour lines below the drops. The temperature contour lines are all straight at the beginning of the simulations, and the migration of drops will then change those lines surrounding the drops. For smaller values of $\gamma$, the contour lines soon become straight again after drops pass, but not for those larger values of $\gamma$. At $t=29$, the contour line near the drop-starting point of the $\gamma=0.25$ run $(z=6.0)$ has already become straight again [Fig. 27(a)], but that of the $\gamma=4.0$ run does not restraighten until $t=74$ [Fig. 28(e)]. When $\gamma$ is larger, the specific heat of the drop is also higher, which means that the background liquid will consume more energy to heat the drop up. As a result, when $\gamma$ is larger, it will take more time for the temperature field below the drop to re-establish the originally constant temperature gradient.

Larger specific heats also mean that the temperature fields inside the drops are less influenced by the temperature fields of the background liquids, so the temperature differences along the interfaces are not so dramatic (e.g., Fig. 29), and accordingly the drops move more slowly (Fig. 30).

\section{THE INFLUENCE OF INITIAL CONDITIONS}

Since many experimental studies related to thermocapillary migrations involve transient phenomena, it is worthwhile to investigate the influence of initial conditions. Normally, people will inject the drop from a container with constant temperature into the bottom of the experimental field. If the injecting and releasing processes of the drop are very short, it is reasonable to assume the initial temperature inside the drop is a constant.

In this section, we consider four runs with the same set of parameters: $\operatorname{Re}_{1}=1, \mathrm{Ma}_{1}=100, \alpha=1, \beta=0.5, \xi=1$, and $\gamma$ $=1$. Most initial conditions remain the same as those before, and the only difference is the initial temperature field inside the drop:

$$
\begin{aligned}
& \text { run A: }\left.\bar{T}\right|_{\bar{t}=0}=T_{0} /(|\nabla T| R)-1, \\
& \text { run B: }\left.\bar{T}\right|_{\bar{t}=0}=T_{0} /(|\nabla T| R), \\
& \text { run C: }\left.\bar{T}\right|_{\bar{t}=0}=T_{0} /(|\nabla T| R)+1, \\
& \text { run D: }\left.\bar{T}\right|_{\bar{t}=0}=T_{0} /(|\nabla T| R)+z / R .
\end{aligned}
$$

It is clear that run D still starts from the classical initial condition [see Eq. (26)], while the initial drop temperatures of Runs A, B, and C are constant. Since we define $z=0$ at the drop center, the initial drop temperatures of runs $\mathrm{A}, \mathrm{B}$, and $\mathrm{C}$ are actually the initial temperatures at the bottom, in the center, and on the top of the drop in run $D$.

It is predictable that the initial conditions of our simulations will not affect the final steady motion since all nondi-

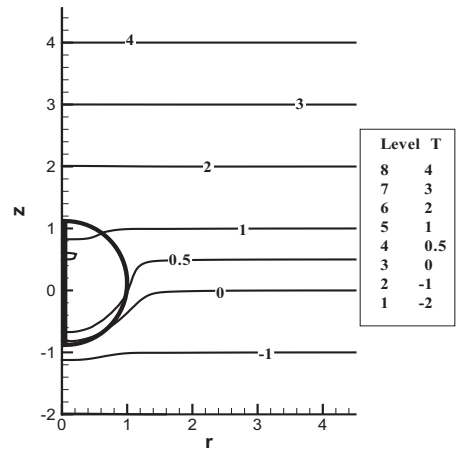

(a) $t=2$

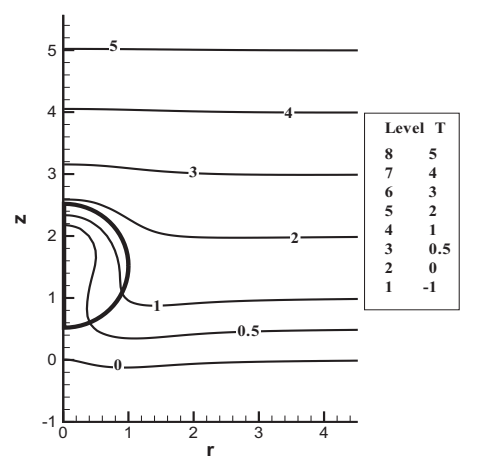

(b) $\mathrm{t}=20$

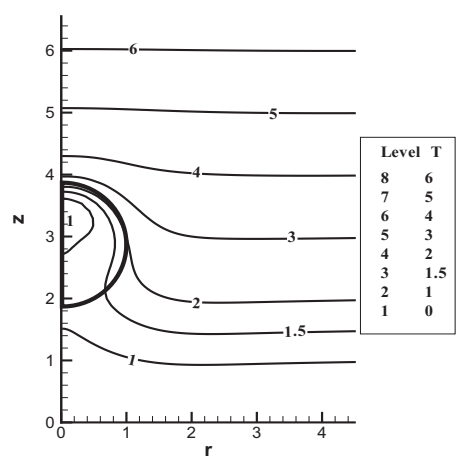

(c) $\mathrm{t}=40$

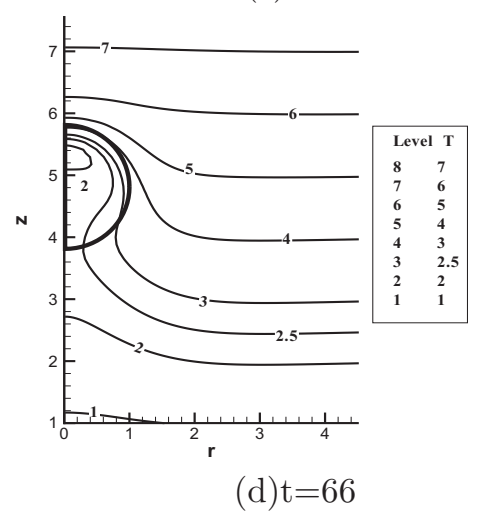

FIG. 33. The isotherm plots of Run C.

mensional numbers are the same. All temperature fields look like the same after $T=40$ (see the last two plots of Figs. 31-34).

There is obvious difference in the time evolution of the migration speeds of four runs (Fig. 35). Because the initial temperature variances of the three new runs (Runs A-C) 


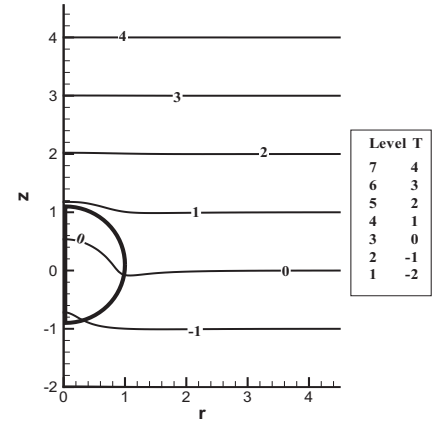

(a) $\mathrm{t}=2$

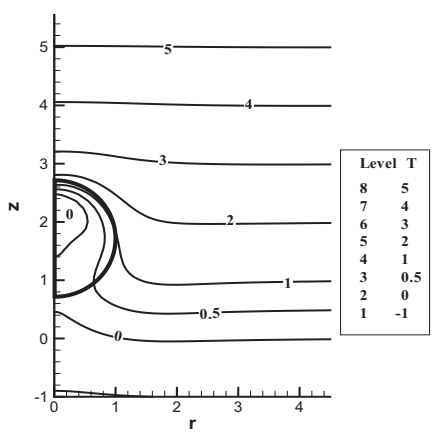

(b) $\mathrm{t}=20$

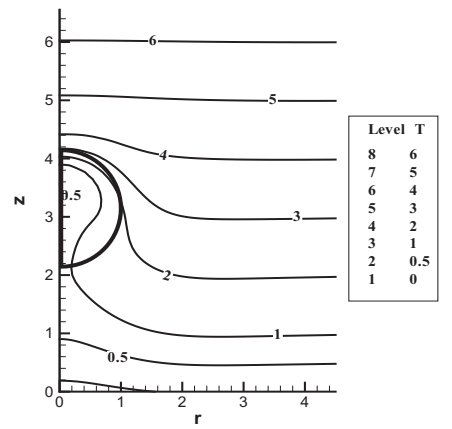

(c) $\mathrm{t}=40$

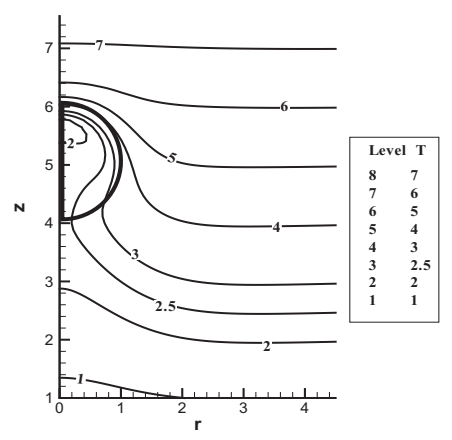

(d) $\mathrm{t}=66$

FIG. 34. The isotherm plots of Run D.

along the interface are much smaller than that of the traditional one (run D), the new runs show much smaller fluctuations in migration velocities at the beginning of the simulations.

It should be noticed that the temperature contours of the background liquid of run A have quite similar shapes throughout the simulation (Fig. 31), which means that the driving force of the drop is almost a constant. Hence, run A has the smallest fluctuation in the time-speed plot (Fig. 35).

Although the overshoot phenomenon in migration veloc-

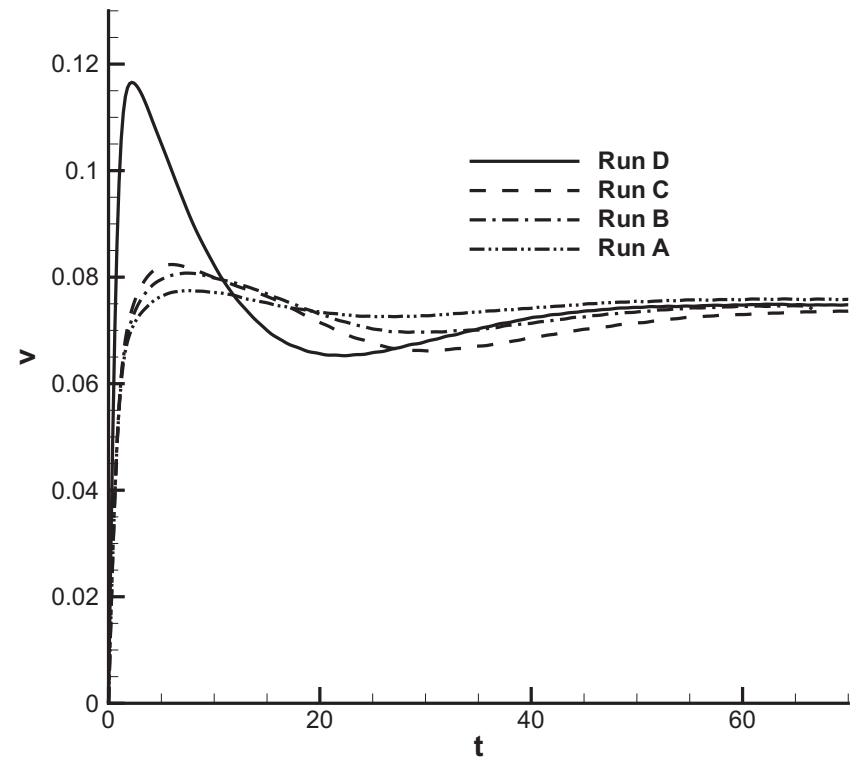

FIG. 35. Time evolutions of drop migration velocities with four different initial conditions.

ity has been reported several times in previous numerical studies, and there is some real physical mechanism behind this phenomenon as discussed earlier in this paper, so far as we know, there have not been any experimental publications claiming to have observed this phenomenon. Hence, maybe the new initial conditions in this subsection are more suitable to be adopted to explain the results from experiments in the future.

\section{CONCLUSIONS}

In this paper, we numerically investigate the thermocapillary migration phenomena of nondeformable spherical drops. To sum up, we can conclude the following.

- The values of $\mathrm{Ma}_{1}$ and $\mathrm{Ma}_{2}$ (or $\beta$ ) have obvious influence on the velocity of the drop. Large Ma number can significantly change the temperature distribution on the interface of the drop, resulting in the undulation of the drop velocity.

- $\mathrm{Re}_{1}$ has direct impact on the migrating process, while it has almost no impact on the final velocity. When $\mathrm{Ma}_{1}$ is fairly large, the increase in $\mathrm{Re}_{1}$ leads to slightly bigger final velocity.

- $\operatorname{Re}_{2}$ or $\alpha$ is the most important factor in our study because it has obvious influence on both migrating velocity and migrating process, and the enlargement of the drop speed caused by bigger $\mathrm{Re}_{2}$ is higher than linear growth.

The changes on the value of $\xi$ and $\gamma$ combined with the variance of four other parameters $\left(\mathrm{Re}_{1}, \mathrm{Ma}_{1}, \alpha, \beta\right)$ have not been fully discussed to avoid the lengthiness of this paper. In the future, before starting to work on the real threedimensional migration of the drop and the interaction 
between multidrops, we need a thorough investigation on the situations associated with more possible values of the six nondimensional numbers discussed in this paper, and also longer simulation times to reach the final steady states in all cases.

\section{ACKNOWLEDGMENTS}

This project is supported by NSF of China (Contract Nos. G10502054 and G10432060) and the Chinese Academy of Sciences Innovation Program. We thank Professor Gretar Tryggvason for clearing some doubts on the numerical scheme.

${ }^{1}$ G. K. Batchelor, An Introduction to Fluid Dynamics (Cambridge, New York, 1990).

${ }^{2}$ N. O. Young, J. S. Goldstein, and M. J. Block, "The motion of bubbles in a vertical temperature gradient," J. Fluid Mech. 6, 350 (1959).

${ }^{3}$ R. S. Subramanian, "Slow migration of a gas bubble in a thermal gradient," AIChE J. 27, 646 (1981).

${ }^{4}$ R. S. Subramanian, "Thermocapillary migration of bubbles and droplets," Adv. Space Res. 3, 145 (1983).

${ }^{5}$ R. Balasubramaniam and R. S. Subramanian, "Thermocapillary bubble migration thermal boundary layers for large Marangoni numbers," Int. J. Multiphase Flow 22, 593 (1996).

${ }^{6}$ R. Balasubramaniam and R. S. Subramanian, "The migration of a drop in a uniform temperature gradient at large Marangoni numbers," Phys. Fluids 12, 733 (2000).

${ }^{7}$ M. Hähnel, V. Delitzsch, and H. Eckelmann, "The motion of droplets in a vertical temperature gradient," Phys. Fluids A 1, 1460 (1989).

${ }^{8} \mathrm{~N}$. Rashidnia and R. Balasubramaniam, "Thermocapillary migration of liquid droplets in a temperature gradient in a density matched system," Exp. Fluids 11, 167 (1991).

${ }^{9}$ Y. S. Chen, Y. L. Lu, Y. M. Yang, and J. R. Maa, "Surfactant effects on the motion of a droplet in thermocapillary migration," Indian J. Pure Appl. Math. 23, 325 (1997).

${ }^{10}$ J. C. Xie, H. Lin, J. H. Han, X. Q. Dong, W. R. Hu, A. Hirata, and M. Sakurai, "Experimental investigation on Marangoni drop migrations using drop shaft facility," Int. J. Heat Mass Transfer 41, 2077 (1998).

${ }^{11}$ J. C. Xie, H. Lin, P. Zhang, F. Liu, and W. R. Hu, "Experimental investigation on thermocapillary drop migration at large Marangoni number in reduced gravity,” J. Colloid Interface Sci. 285, 737 (2005).

${ }^{12}$ J. Szymczyk and J. Siekmann, "Numerical calculation of the thermocap- illary motion of a bubble under microgravity," Chem. Eng. Commun. 69, 129 (1988)

${ }^{13}$ N. Shankar and R. S. Subramanian, "The stokes motion of a gas bubble due to interfacial tension gradients at low to moderate Marangoni numbers," J. Colloid Interface Sci. 123, 512 (1988).

${ }^{14}$ R. Balasubramaniam and J. E. Lavery, "Numerical simulation of thermocapillary bubble migration under microgravity for large Reynolds and Marangoni numbers," Numer. Heat Transfer, Part A 16, 175 (1989).

${ }^{15}$ X. J. Ma, R. Balasubramaniam, and R. S. Subramanian, "Numerical simulation of thermocapillary drop motion with internal circulation," Numer. Heat Transfer, Part A 35, 291 (1999).

${ }^{16} \mathrm{H}$. Haj-Hariri, Q. Shi, and A. Borhan, "Thermocapillary motion of deformable drops at finite Reynolds and Marangoni numbers," Phys. Fluids 9, 845 (1997).

${ }^{17}$ S. Nas and G. Tryggvason, "Thermocapillary interaction of two bubbles or drops," Int. J. Multiphase Flow 29, 1117 (2003).

${ }^{18}$ S. Nas, M. Muradoglu, and G. Tryggvason, "Pattern formation of drops in thermocapillary migration," Int. J. Heat Mass Transfer 49, 2265 (2006).

${ }^{19}$ R. Balasubramaniam, C. E. Lacy, G. Woniak, and R. S. Subramanian, "Thermocapillary migration of bubbles and drops at moderate values of the Marangoni number in reduced gravity," Phys. Fluids 8, 872 (1996).

${ }^{20}$ P. H. Hadland, R. Balasubramaniam, G. Wozniak, and R. S. Subramanian, "Thermocapillary migration of bubbles and drops at moderate to large Marangoni number and moderate Reynolds number in reduced gravity," Exp. Fluids 26, 24 (1999).

${ }^{21}$ R. B. Bird, W. E. Stewart, and E. N. Lightfoot, Transport Phenomena (Wiley, New York, 2002).

${ }^{22}$ L. G. Leal, Laminar Flow and Convective Transport Processes (Butterworth-Heinemann, Boston, 1992).

${ }^{23}$ P. Gao, Z. H. Yin, and W. R. Hu, "Thermocapillary motion of droplets at large Marangoni numbers," Adv. Space Res. 41, 2101 (2008).

${ }^{24}$ S. O. Unverdi and G. Tryggvason, "A front-tracking method for viscous incompressible flows," J. Comput. Phys. 100, 25 (1992).

${ }^{25}$ G. Tryggvason, B. Bunner, A. Esmaeeli, D. Juric, N. Al-Rawahi, W. Tauber, J. Han, S. Nas, and Y.-J. Jan, "A front-tracking method for the computations of multiphase flow," J. Comput. Phys. 196, 708 (2001).

${ }^{26}$ C. S. Peskin, "Numerical analysis of blood flow in the heart," J. Comput. Phys. 25, 220 (1977).

${ }^{27}$ D. L. R. Oliver and K. J. DeWitt, "Transient motion of a gas bubble in a thermal gradient in low gravity," J. Colloid Interface Sci. 164, 263 (1994).

${ }^{28}$ M. D. Levan, "Motion of a droplet with a Newtonian interface," J. Colloid Interface Sci. 83, 11 (1981).

${ }^{29}$ S. W. J. Welch, "Transient thermocapillary migration of deformable bubbles,” J. Colloid Interface Sci. 208, 500 (1998). 